\title{
Vortex Deconfinement in the $X Y$ Model with a Magnetic Field
}

\author{
H. A. Fertig( ${ }^{(1)}$ and Kingshuk Majumdar ${ }^{(2)}$ \\ (1) Department of Physics and Astronomy, University of Kentucky, Lexington, KY 40506-0055; \\ (2) Department of Physics, Berea College, Berea, KY 40404
}

(June 24, 2021)

\begin{abstract}
We study vortex unbinding for the classical two-dimensional $X Y$ model in a magnetic field on square and triangular lattices. A renormalization group analysis combined with duality in the model shows that at high temperature and high field, the vortices unbind as the magnetic field is lowered in a twostep process: strings of overturned spins first proliferate and then vortices unbind. The transitions are highly continuous but are not of the Kosterlitz-Thouless type. The unbound vortex fixed point is shown to inherit properties of the underlying lattice, in particular containing a set of nodal lines that reflect the lattice symmetry.
\end{abstract}

PACS numbers: 64.60.Ak, 73.43.Cd, 74.76.-w, 75.10.Hk

\section{INTRODUCTION}

Topological defects play a crucial role in a host of phenomena in condensed matter systems ${ }^{1}$. Among these are Kosterlitz-Thouless (KT) transitions ${ }^{2}$, in which vortexlike defects in a two dimensional system unbind above some critical temperature. Kosterlitz-Thouless transitions are important for understanding disordering in planar $(X Y)$ magnets, destruction of superfluidity and superconductivity in thin films, melting of two dimensional crystals, and transitions among different liquid crystal states $^{1}$. Vortex unbinding is related to the roughening transition, in which the interface between two materials fluctuates without bound above a critical temperature ${ }^{3}$. They are important for understanding ground state properties of $1+1$ dimensional quantum systems (Luttinger liquids $)^{4}$. They have also recently found relevance in understanding the states of stripe systems in high temperature superconductors and quantum Hall systems ${ }^{5,6}$. The $X Y$ ferromagnet is often considered the paradigm of all these systems, largely because of the (somewhat misleading) simplicity of its Hamiltonian.

Much less attention has been paid to what becomes of the vortex unbinding transition in the presence of a symmetry-breaking field, such as a magnetic field tending to align the $X Y$ spins along some particular direction, in spite of the fact that most of the systems discussed above have realizations in which such symmetry-breaking fields are present. One major reason for this is that the standard KT analysis breaks down under the most interesting circumstances when a symmetry breaking field is present. Usually, one assesses whether vortices may be incorporated into a simple theoretical description of a system by renormalization of the parameters of the Hamiltonian. This is possible when the vortices are bound tightly into pairs of vanishing net vorticity. In the renormalization group (RG) approach to the KT transition, the paired state is signaled by a flow in which the vortex fugacity, $e^{-E_{c} / k_{B} T} \equiv y / 2$, with $E_{c}$ the core energy of a vortex, vanishes. The interpretation of this is that an effective long-wavelength theory of the system may be constructed with vanishing vortex density $(y=0)$; the vortex degrees of freedom are irrelevant in the renormalization group sense $^{7}$.

For small values of $y$ and above the Kosterlitz-Thouless transition temperature $T_{K T}$, one finds that $y$ grows rather than shrinks, signaling the relevance of vortices in the system, so that a theory without vortices cannot be qualitatively correct. This sharp change in behavior is accompanied by an essential singularity in the freeenergy, so that vortex unbinding constitutes a continuous phase transition. Because the RG flows contain a set of simple attractive fixed points (the Hamiltonians with $y=0$ to which the low temperature microscopic Hamiltonians flow under the RG), thermodynamic properties of the transition may be computed ${ }^{1,2}$.

For many systems with symmetry-breaking fields, the existence of this set of stable fixed points is lost ${ }^{8}$. A prototype of this - and the system we focus on in this paper - is the $X Y$ system with a magnetic field. It is defined by the Hamiltonian

$$
\mathcal{H}_{X Y} / k_{B} T=-K \sum_{<\mathbf{r}, \mathbf{r}^{\prime}>} \cos \left(\theta_{\mathbf{r}}-\theta_{\mathbf{r}^{\prime}}\right)-h \sum_{\mathbf{r}} \cos \theta_{\mathbf{r}} .
$$

In Eq. $1, \theta_{\mathbf{r}}$ represents the angle of a planar spin at location $\mathbf{r}, K$ is an exchange coupling between nearest neighbor spins, and $h$ is the magnitude of an effective magnetic field tending to align the spins along the $\hat{\mathbf{x}}$ axis. For convenience from now on we will choose our units of energy so that $k_{B} T=1$. A perturbative RG analysis ${ }^{8}$ when applied to this system shows that either the symmetry-breaking term in the Hamiltonian or the vortex fugacity, or both, are relevant even when they are initially very small. The absence of a stable fixed point means this perturbative analysis cannot provide us with a simple Hamiltonian to describe the state of the system. This has been interpreted to mean that there is no phase transition, and presumably no possibility of vortex unbinding, when a symmetry-breaking field is present (see, for example, Chap. 2 of Ref. 1). In this paper, we will describe a new analysis that suggests that while the first part of this interpretation (no phase transition) is at some level true, the second part (no vortex unbinding) is not: 
we will develop a RG description that allows for vortex unbinding, but with a fixed point structure that remarkably avoids the free energy singularity usually associated with a phase transition. Some of the results discussed here were described elsewhere ${ }^{9}$. In this paper we provide details of those calculations, and extend them to show how the underlying lattice symmetry may be incorporated into the model.

An important perspective on why unbinding in the $X Y$ ferromagnet is different in the presence of a magnetic field than without it comes from examining the interaction of a single vortex-antivortex pair. Without symmetry breaking, this is well known to be a slow, logarithmic potential with respect to pair separation. This arises because the lowest energy spin configuration, subject to the constraint that the system contains a vortex and an antivortex, approaches the ferromagnetic ground state slowly as one moves away from the pair. Such a configuration is energetically expensive for $h>0$ since it involves many spins tilted away from the direction specified by the field; the system instead forms a string of overturned spins connecting the vortex-antivortex pair ${ }^{10}$. The rotation of the spins through $2 \pi$ as one moves through the string is essentially identical to a kink soliton of the sine-Gordon mode ${ }^{11}$, carrying a characteristic energy per unit length proportional to $\sqrt{K h}$. Thus, the potential energy for a pair of vortices increases linearly with separation. Such linear potentials arise in the context of strong interactions, and leads to confinement ${ }^{12}$, a situation in which individual particles (quarks for strong interactions, vortices in the $X Y$ model) do not appear in isolation. From this analogy one might conclude there is no unbound vortex state of this system.

The difficulty with this argument is that it ignores the statistical mechanics of the string connecting the vortices. At high temperatures, one must include entropy in the free energy of the string connecting a single vortex-antivortex pair. The number of configurations for a string grows exponentially ${ }^{13}$ with its length $L$, so that at high temperature a string cannot bind a single vortex-antivortex pair. Moreover, the string tension - its energy per unit length - is renormalized downward by small breaks (vortex-antivortex pairs), which may occur along its length. Finally, at high temperature the $X Y$ system will be flooded with vortices and antivortices, so that the distance between neighboring pairs will be of the same order as the pair sizes themselves. This situation is highly reminiscent of what is expected to occur in a quark-gluon plasma, in which quarks are deconfined ${ }^{12}$. Deconfinement at high temperature is clearly also a possibility for vortices in the $X Y$ system. To decide if and when this occurs, we will develop a model that deals $d i$ rectly with the strings, and contains a parameter which we will identify with the string tension. This string tension can be driven to zero when renormalized by fluctuations - signaling an unbinding transition for the vortices.

Before beginning our technical discussion, we summarize our results. We develop an effective model for this system, focusing on a representation in which the string degrees of freedom are explicit. The model has a dual form, which may be interpreted as a solid-on-solid model with screw dislocations. The resulting low energy excitations contain both open and closed domain walls. The endpoints of the domain walls - the screw dislocations are degrees of freedom dual to the vortices. We initially focus on the states of these dislocations, and then use the duality to draw conclusions about the allowed states of the vortices. The perturbative RG analysis we follow is valid in the large $h$ and small $y$ limit. It generates a parameter $\rho$, which represents the energy per unit distance for separating two endpoints of a single open domain wall, keeping the length of the domain wall fixed. The RG equations indicate that $\rho$ may flow to zero, or may flow to a finite value; we interpret the latter as a bound dislocation state. The $\rho=0$ fixed point is accessible when the domain walls are rough, i.e., when they are unbounded in size and percolate through the system. Since arbitrarily large domain walls may be cut open with endpoints as far apart as we like without extra energy cost at the $\rho=0$ fixed point, we identify this as an unbound dislocation phase.

In the RG flows, the $\rho=0$ fixed point appears at the end of a line of fixed points with $\rho \geq 0$, so that the flows accumulate at the $\rho=0$ for the unbound phase. The string tension $\rho$ grows continuously from zero when the microscopic parameters of the Hamiltonian cross into the values corresponding to the bound dislocation phase. For fixed $K$ and $E_{c}$, bound dislocations occur for $h$ smaller than some critical value.

An important observation is that there is no relevant direction in the parameter space of the Hamiltonian leading away from the unbound dislocation fixed point. This is a remarkable result: in the absence of a relevant direction, there is no mechanism by which the effective free energy can accumulate a singularity in the RG as one integrates out short distance scales. With no free energy singularity, one does not expect to find singularities in any thermodynamic quantities for the system. Nevertheless, we can sharply distinguish the bound and unbound vortex phases, for example by measuring the diffusion constant for vortices, or (equivalently) measuring fluctuations in the vortex dipole moment of the system. Recent Langevin dynamics simulations ${ }^{14}$ focusing on the latter quantity have confirmed the basic results of the RG studies presented here; we will discuss these in more detail below. The important lesson at this point is that vortex deconfinement in this system is not a phase transition in the usual sense. One can distinguish the bound and unbound vortex phases through transport properties, correlation functions, or statistics of specific fluctuations in the system. But they cannot be distinguished by qualitative differences in quantities that may be expressed as derivatives of the free energy; i.e., thermodynamic quantities. The possibility of such unusual transitions has been noted in rigorous studies of phase transitions ${ }^{15}$, but to our knowledge this is the first concrete example of such 
behavior.

The unbound dislocation fixed point, we shall see, has a unique signature: it contains one or more lines of zero energy modes in the Brillouin zone. Our earlier analysis ${ }^{9}$ focused on the case of a single such nodal line. This breaks the discrete rotational symmetry of the lattice, and we will see it arises from using a particular choice of gauge which leads to approximations breaking the symmetry. We will show that the underlying symmetry of the lattice may be retained without changing any of the qualitative results. In the case of a square lattice, the fixed point contains two nodal lines, and for a triangular lattice it contains three. The number of nodal lines is determined by the number of independent directions a domain wall may exit from a dislocation; it is essentially the number of distinguishably different types of dislocations the system supports. We note that these fixed points might be regarded as a classical analog of similar Hamiltonians arising in the context of quantum spin models with ring exchange ${ }^{16}$.

To understand what all this implies about vortex unbinding, we must re-express the partition function in its dual form. The dual Hamiltonian we will see is identical to the dislocation Hamiltonian, provided we exchange $E_{c} \leftrightarrow 1 / 2 h$ and substitute $K \rightarrow 1 / 4 \pi^{2} K$. Since the unbound dislocation phase occurs for large $E_{c}$ and small $1 / 2 h$, we expect unbound vortices can be found at large $1 / 2 h$ and small $E_{c}$. That an unbound vortex phase does not occur for large values of $E_{c}$ shows how different the transition is from the $\mathrm{KT}$ phenomenology, and also is consistent with the failure of perturbative RG calculations ${ }^{8}$ with small $y=e^{-E_{c}}$ (i.e., large $E_{c}$ ) to capture the transition. Since dislocation unbinding occurs at a finite value of $1 / 2 h$, dislocation unbinding and vortex unbinding cannot be the same transition. This tells us there must be an intermediate phase in which both dislocations and vortices are bound. The nature of this phase follows from the observation that domain walls are rough even in the bound dislocation phase. At long wavelengths, we can ignore the dislocations, so one is effectively in the rough (high temperature) phase of a solid-on-solid model. This is dual to a bound (low temperature) phase of logarithmically interacting vortices ${ }^{3}$, so we identify the large $E_{c}$, $1 / 2 h$ limit of the parameters as a logarithmically bound dislocation phase. From the duality, we expect this implies the vortices will also be logarithmically bound in this region of parameters. Physically, we can understand this by recognizing the domain walls in the dislocation representation are dual to the strings in the vortex representation, and both are proliferated for these parameters. Proliferated strings do not linearly confine the vortices, but they can (and do) upwardly renormalize the logarithmic interactions between them, enough so that the vortices may be bound together even if $T>T_{K T}$.

Finally, one may ask what the unbound dislocation phase looks like when expressed in terms of the vortex degrees of freedom. Since this occurs for very large values of $h$ and $E_{c}$, the bare string tension between vortices is

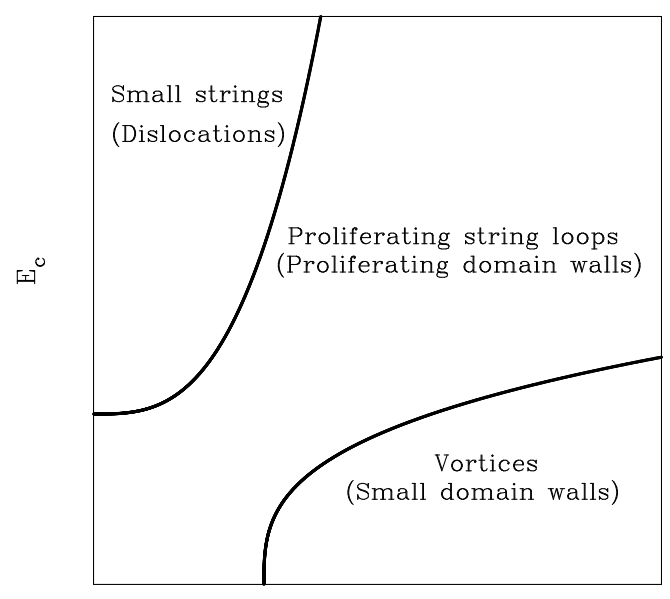

$1 / 2 \mathrm{~h}$

FIG. 1. Schematic phase diagram for $X Y$ model in a magnetic field for $K \approx 1 / 2 \pi$. Upper left corner represents linearly confined phase (unbound screw dislocations in dual representation). Middle phase contains proliferated loops in both descriptions and vortices (dislocations) are logarithmically bound. Lower right corner contains unbound vortices (linearly confined dislocations).

very large and the vortices are dilute. In this case it is clear that the vortices are linearly confined. Thus we identify the highest temperature phase of the dislocations - a deconfined phase - with the lowest temperature phase of the vortices - a linearly confined phase.

All these considerations suggest that for large $E_{c}$ and/or small $h$, there are three phases for the vortices: a linearly confined phase, a logarithmically confined phase, and a deconfined phase. This is illustrated in Fig. 1, which is the simplest phase diagram one may draw consistent with the perturbative RG analysis.

We are led to a picture in which vortices deconfine in a two-step process: as fluctuations increase, the strings connecting them proliferate, but vortex-antivortex pairs remain bound by a residual logarithmic attraction; at still higher levels of fluctuations the closed string loops may break open, and the vortices deconfine. We note that simulations strongly support the existence of two different bound phases, as well as the unbound vortex phase $^{14}$.

This article is organized as follows. We begin Section II by expressing the system as a Villain model, and then formulate the model as a continuum theory. In Section III we develop the RG calculations and show how the unusual fixed point structure emerges. Section IV discusses more careful formulations that respect the lattice symmetry for square and triangular lattices. Section V contains discussion of the numerical integration of the RG equations, and we conclude with a summary in Section VI. Two Appendices provide further details of the calculations. 


\section{DERIVATION AND INTERPRETATION OF THE MODEL}

\section{A. Representation in Terms of Domain Walls and Screw Dislocations}

In this section, we present the derivation of the model we will be considering. Many of the steps we take may be found in Ref. 8, and indeed our final result is equivalent to theirs, although the representation on which we finally perform our RG analysis is considerably different, leading to different results. In any case, for completeness we present the derivation in its entirety.

The partition function for the $X Y$ model may be expressed as $\mathcal{Z}_{X Y}=\int \mathcal{D} \theta e^{-\mathcal{H}_{X Y}}$. Because of the cosines appearing in the exponent, it is very difficult to make progress working directly with this Hamiltonian. Since we are interested in phases and long wavelength properties of the system, we can replace the model with any other that contains the same symmetries and retain the correct physics. A particularly useful model to adopt is the Villain $\operatorname{model}^{17}$, in which one makes the replacement

$$
e^{C \cos \theta} \rightarrow \sum_{m=-\infty}^{\infty} e^{-C(\theta-2 \pi m)^{2} / 2}
$$

whenever a cosine appears in the exponent. $m$ above is an integer variable, and the important observation is that the weight as a function of $\theta$ has period $2 \pi$ on both sides of Eq. 2. The replacement of a cosine in the exponent with a quadratic form allows us to make some progress. Applying this to $\mathcal{Z}_{X Y}$, we are led to the replacement

$$
\begin{aligned}
\mathcal{Z}_{X Y} \rightarrow \mathcal{Z}_{V M} & \\
=\sum_{m_{\mathbf{r}, \mathbf{r}^{\prime}}} \sum_{n_{\mathbf{r}}} \int \mathcal{D} \theta \exp & {\left[-\frac{K}{2} \sum_{\left\langle\mathbf{r}, \mathbf{r}^{\prime}\right\rangle}\left(\theta_{\mathbf{r}}-\theta_{\mathbf{r}^{\prime}}-2 \pi m_{\mathbf{r}, \mathbf{r}^{\prime}}\right)^{2}\right.} \\
& \left.-\frac{h}{2}\left(\theta_{\mathbf{r}}-2 \pi n_{\mathbf{r}}\right)^{2}\right]
\end{aligned}
$$

The functional integral over the $\theta$ field may be performed with the help of the Poisson resummation formula. This states that for a function $g(m)$ summed over an integer field $m$,

$$
\sum_{m=-\infty}^{\infty} g(m)=\sum_{n=-\infty}^{\infty} \int_{-\infty}^{\infty} d \phi g(\phi) e^{-2 \pi i n \phi} .
$$

Applying this to both integer fields in Eq. 3 and shifting variables, we arrive at the expression

$$
\begin{aligned}
\mathcal{Z}_{V M} & =\sum_{S_{\mathbf{r} \mathbf{r}^{\prime}}} \sum_{T_{\mathbf{r}}} \int \mathcal{D} \theta \int \mathcal{D} \phi \int \mathcal{D} \psi \\
\times \exp & {\left[-2 \pi^{2} K \sum_{\left\langle\mathbf{r}, \mathbf{r}^{\prime}\right\rangle} \phi_{\mathbf{r}, \mathbf{r}^{\prime}}^{2}-2 \pi^{2} h \sum_{\mathbf{r}} \psi_{\mathbf{r}}^{2}\right.} \\
& \left.-2 \pi i \sum_{\left\langle\mathbf{r}, \mathbf{r}^{\prime}\right\rangle} S_{\mathbf{r r}^{\prime}} \phi_{\mathbf{r}, \mathbf{r}^{\prime}}\right]
\end{aligned}
$$

$$
\begin{aligned}
\times \exp & {\left[-2 \pi i \sum_{\mathbf{r}} T_{\mathbf{r}} \psi_{\mathbf{r}}-i \sum_{\left\langle\mathbf{r}, \mathbf{r}^{\prime}\right\rangle}\left(\theta_{\mathbf{r}}-\theta_{\mathbf{r}^{\prime}}\right) S_{\mathbf{r r}^{\prime}}\right.} \\
& \left.-i \sum_{\mathbf{r}} \theta_{\mathbf{r}} T_{\mathbf{r}}\right],
\end{aligned}
$$

where $S_{\mathbf{r r}^{\prime}}$ and $T_{\mathbf{r}}$ are the integer fields arising from the Poisson resummations. The continuous fields in Eq. 4 may now all be integrated out. We focus first on the angular variables $\theta$, for which the integration produces a product of $\delta$-functions. These may be conveniently written if we re-express the bond integer field $S_{\mathbf{r r}^{\prime}}$ as a vector field $\mathbf{S}(\mathbf{r})$, with components $S_{i}(\mathbf{r})=S_{\mathbf{r}, \mathbf{r}+\boldsymbol{\Delta}_{i}}$, where $\boldsymbol{\Delta}_{i}$ are vectors denoting nearest neighbor bonds in the "positive" direction (For example, on a square lattice one may take $\boldsymbol{\Delta}_{1}=a_{0} \hat{\mathbf{y}}, \boldsymbol{\Delta}_{2}=a_{0} \hat{\mathbf{x}}$.). It is important to note that the dimensionality of $\mathbf{S}$ is not set by the (two-) dimensionality of the lattice, but rather is given by (half) the coordination number; for example, in the triangular lattice discussed below, $\mathbf{S}$ is a three-dimensional vector field. Whatever its dimensionality, we can define a divergence,

$$
\nabla \cdot \mathbf{S}(\mathbf{r}) \equiv \sum_{i}\left[S_{i}(\mathbf{r})-S_{i}\left(\mathbf{r}-\boldsymbol{\Delta}_{i}\right)\right] .
$$

The second line of Eq. 4 can now be rearranged to read

$$
\times \exp \left[-2 \pi i \sum_{\mathbf{r}} T_{\mathbf{r}} \psi_{\mathbf{r}}-i \sum_{\mathbf{r}} \theta_{\mathbf{r}}\left[T_{\mathbf{r}}-\nabla \cdot \mathbf{S}(\mathbf{r})\right]\right],
$$

from which it is now clear that the angular integration

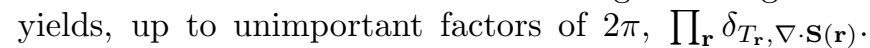
Substituting this into Eq. 4, performing the remaining continuous integrals and using the $\delta$ functions to eliminate the $T$ sum, we arrive at the remarkably simple form (ignoring unimportant prefactors) $\mathcal{Z}_{V M}=\sum_{\mathrm{S}} e^{-\mathcal{H}_{V M}}$, with

$$
\mathcal{H}_{\mathrm{VM}}=\frac{1}{2 K} \sum_{\mathbf{r}}|\mathbf{S}(\mathbf{r})|^{2}+\frac{1}{2 h} \sum_{\mathbf{r}}(\nabla \cdot \mathbf{S}(\mathbf{r}))^{2} .
$$

In the limit $h \rightarrow 0$, the configurations entering the partition sum must satisfy $\nabla \cdot \mathbf{S}(\mathbf{r})=0$, which implies ${ }^{18}$ one may write $\mathbf{S}$ as a two dimensional curl of an integer function $n, \mathbf{S}(\mathbf{r})=\hat{\mathbf{z}} \times \nabla n(\mathbf{R})$, where $n$ should be understood as residing on sites of the dual lattice, $\mathbf{R}$ (see Fig. 2). Thus we get an effective Hamiltonian for the $h \rightarrow 0$ limit

$$
\mathcal{H}(h \rightarrow 0)_{\mathrm{VM}} \equiv \mathcal{H}_{\mathrm{DG}}=\frac{1}{2 K} \sum_{\mathbf{R}}|\nabla n(\mathbf{R})|^{2} .
$$

$\mathcal{H}_{\mathrm{DG}}$ is the "discrete Gaussian model", which is one of the simplest solid-on-solid models of an interface ${ }^{19}$. This is well-known to be dual to the two dimensional Coulomb gas, and so undergoes a Kosterlitz-Thouless transition; in the context of a solid-on-solid model, it is a roughening transition $^{3}$. It is helpful to recognize what is going on in the roughening transition in terms of the original $\mathbf{S}$ variables before returning to the case $h>0$. Since $n(\mathbf{R})$ represents the height of fluctuating columns in an interface, we recognize that bonds for which $\mathbf{S}(\mathbf{r})=\hat{\mathbf{z}} \times \nabla n(\mathbf{R}) \neq 0$ 
actually represent domain walls between regions of different heights. Note that the condition $\nabla \cdot \mathbf{S}(\mathbf{r})=0$ guarantees that the domain walls must be closed. The important point is that the roughening transition represents the temperature above which such closed domain walls proliferate, i.e., percolate through the system. When expressed in terms of the Coulomb gas (i.e., vortex) model, the high temperature, rough phase corresponds to the low temperature, bound pair phase.

Returning to the $h>0$ phase, we see that Eq. 5 allows us to work directly with the domain wall degrees of freedom. The fact that we allow configurations with $\nabla \cdot \mathbf{S}(\mathbf{r}) \neq 0$ means that we are allowing open as well as closed domain walls. In fact, locations for which $\nabla \cdot \mathbf{S}(\mathbf{r}) \neq 0$ locate points where a domain wall comes to an end. This is precisely the situation one has when an interface contains screw dislocations: locations for $\nabla \cdot \mathbf{S}(\mathbf{r}) \neq 0$ define the centers of these screw dislocations, and from the second term of $\mathcal{H}_{\mathrm{VM}}$ we identify the core energy of the dislocation as $1 / 2 h$. Remarkably, we have arrived at a theory that involves string-like objects (the domain walls) and vortex-like objects (the screw dislocations), which is very much in line with what we expected from the considerations discussed in the Introduction. We will see that Eq. 5 is essentially the dual of a model in which the string and vortex degrees of freedom are explicit; however, we will need to introduce the vortex core energy before this is apparent.

The above discussion shows that in our model (and its dual) the dislocations (vortices) are endpoints of domain walls (strings), so that when they are in a bound state, basic excitations of the system contain closed loops. We would like a representation of the model that captures this physics, presumably one that is closely related to Eq. 6. Toward this end we need to represent the domain wall degrees of freedom $\mathbf{S}$ as differences. This is most easily developed for the square lattice, so for the rest of this section and the next few, we specialize to this case; the triangular lattice will be dealt with in Section IV B. We start by writing

$$
\begin{aligned}
S_{1}(\mathbf{r}) & =m_{1}\left(\mathbf{R}=\mathbf{r}+\boldsymbol{\Delta}_{\mathbf{1}} / 2-\boldsymbol{\Delta}_{\mathbf{2}} / 2\right) \\
& -m_{1}\left(\mathbf{R}=\mathbf{r}+\boldsymbol{\Delta}_{\mathbf{1}} / 2+\boldsymbol{\Delta}_{\mathbf{2}} / 2\right) \\
S_{2}(\mathbf{r}) & =m_{2}\left(\mathbf{R}=\mathbf{r}+\boldsymbol{\Delta}_{\mathbf{1}} / 2+\boldsymbol{\Delta}_{\mathbf{2}} / 2\right) \\
& -m_{2}\left(\mathbf{R}=\mathbf{r}-\boldsymbol{\Delta}_{\mathbf{1}} / 2+\boldsymbol{\Delta}_{\mathbf{2}} / 2\right)
\end{aligned}
$$

Note that we have located the integer fields $m_{1}, m_{2}$ on the dual lattice sites $\{\mathbf{R}\}$ of the original square lattice. In Sections IV A and IV B, we will see that representations such as Eqs. 7 are convenient for finding fixed points that respect the lattice symmetry. For now, we make the further transformation

$$
\begin{aligned}
& m_{2}(X, Y)=n(X, Y), \\
& m_{1}(X, Y)=n(X, Y)+\sum_{x^{\prime}=x_{0}}^{X-\Delta_{2} / 2} A\left(x^{\prime}, Y\right)
\end{aligned}
$$

where $\mathbf{R}=(X, Y)$ are sites on the dual square lattice, $n$ and $A$ are integer fields, and the sum in the second of

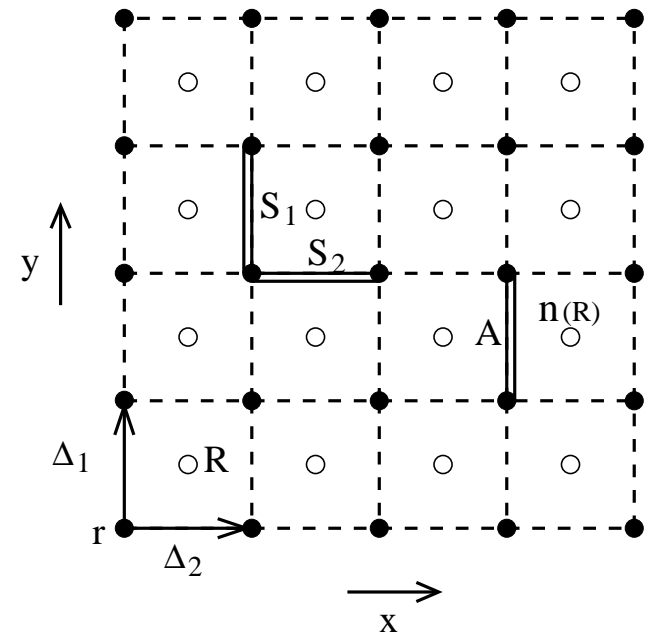

FIG. 2. Real lattice sites (r) (black filled circles) and dual lattice sites (R) (open circles) of a square lattice. $\boldsymbol{\Delta}_{\mathbf{1}}=a_{0} \hat{\mathbf{y}}$ and $\boldsymbol{\Delta}_{\mathbf{2}}=a_{0} \hat{\mathbf{x}}$ are the vectors denoting nearest neighbor bonds. $S_{1}$ and $S_{2}$ are bond integer fields that live on the nearest neighbor bonds as shown. $S_{1}$ and $S_{2}$ can be written in terms of integers $m_{1}$ and $m_{2}$ on the dual sites $\{\mathbf{R}\}$ as defined in Eq. 7. A further transformation (Eq. 8) defines two integer fields $n$ and $A$, where $A$ lives on the vertical bonds and $n$ resides on the dual lattice sites.

Eqs. 8 is along rows in the square array, starting at some reference line $x_{0}$ which can conveniently be chosen as the boundary of the system. If one fixes the values of $n$ and $A$ along this boundary (e.g., $n\left(x_{0}, y\right)=0, A\left(x_{0}, Y\right)=0$ ) it is not hard to see that there is a one-to-one invertible mapping between the fields $\left(S_{1}, S_{2}\right)$ and $(n, A)$, so the new degrees of freedom capture all the domain wall configurations without overcounting them ${ }^{20}$. Notice in this representation it is natural to think of the $n$ field as residing on the dual lattice sites, while the $A$ field resides on the vertical bonds of the original lattice. This is shown in Fig. 2.

We now have the representation

$$
\begin{aligned}
\mathcal{H}_{\mathrm{VM}} & =\frac{1}{2 K} \sum_{\mathbf{r}}\left|(\nabla n)_{\mathbf{r}}+A\left(x, y+\Delta_{1} / 2\right) \hat{\mathbf{x}}\right|^{2} \\
& +\frac{1}{2 h} \sum_{\mathbf{r}}\left[\left(\frac{\partial A}{\partial y}\right)_{\mathbf{r}}\right]^{2} .
\end{aligned}
$$

In this representation, it is clear that the $n$ field allows us to represent configurations with closed domain walls. The $A$ field introduces the open domain wall configurations in two ways: it allows us to directly occupy the vertical bonds with non-zero values, and it allows us to remove the domain wall energy along vertical bonds of closed domain walls - which allows open configurations with horizontal domain wall segments. Fig. 3 illustrates an example of this. 


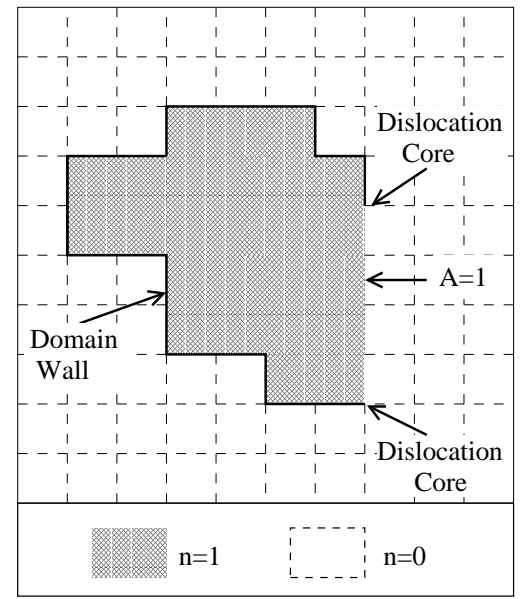

FIG. 3. A low energy configuration involving $A \neq 0$. A region of $n=1$ (hatched squares) is embedded in a surrounding $n=0$ region (white squares); heavy line represents a domain wall. The line segment with $A=1$ cancels the domain wall energy for part of its length, leaving an open domain wall.

\section{B. Vortex Representation}

To explicitly show the duality between dislocations and vortices, as well as to introduce the core energy of the vortices, we need a representation in which the vortex degrees of freedom are explicit. This is accomplished by eliminating $n$ using the Poisson resummation formula, just as in the case $h=0$. The partition sum becomes

$$
\begin{aligned}
\mathcal{Z}_{\mathrm{VM}} & =\sum_{\{n\}} \sum_{\{A\}} \exp \left[-\mathcal{H}_{\mathrm{VM}}[\{n\},\{A\}]\right], \\
& =\sum_{\{A\}} \sum_{\{m\}} \int \mathcal{D} \phi \exp \left[-\mathcal{H}_{\mathrm{VM}}[\{\phi\},\{A\}]\right. \\
& \left.-2 \pi i \sum_{\mathbf{R}} \phi(\mathbf{R}) m(\mathbf{R})\right]
\end{aligned}
$$

The functional integral in Eq. 10 may be carried through if we represent the fields in terms of their Fourier transforms. Writing $\phi(\mathbf{q})=\sum_{\mathbf{R}} e^{i \mathbf{q} \cdot \mathbf{R}} \phi(\mathbf{R})$, with $N$ the number of lattice sites, and similar definitions for $m(\mathbf{q}), n(\mathbf{q})$, and $A(\mathbf{q})$, we have

$$
\begin{aligned}
& \mathcal{Z}_{\mathrm{VM}} \\
& =\sum_{\{A\}} \sum_{\{m\}} \int \mathcal{D} \phi \exp \left[-\frac{1}{2 K N} \sum_{\mathbf{q}}|\mathbf{Q} \phi(\mathbf{q})+A(\mathbf{q}) \hat{\mathbf{x}}|^{2}\right. \\
& -\frac{1}{2 h N} \sum_{\mathbf{q}}\left|Q_{y} A(\mathbf{q})\right|^{2} \\
& \left.-\frac{2 \pi i}{N} \sum_{\mathbf{q}} \phi(\mathbf{q}) m(\mathbf{q})\right] \text {, }
\end{aligned}
$$

where $\mathbf{Q}=\left(Q_{x}, Q_{y}\right)$, and $Q_{x}=1-e^{-i \mathbf{q} \cdot \Delta_{2}}, Q_{y}=1-$ $e^{-i \mathbf{q} \cdot \Delta_{1}}$. Integrating out $\phi$ gives, up to an unimportant prefactor,

$$
\begin{aligned}
\sum_{\{A\}} \sum_{\mathrm{VM}} \exp \{ & -\frac{1}{N} \sum_{\mathbf{q}}\left[\frac{1}{2 K|Q|^{2}}+\frac{1}{2 h}\right]\left|Q_{y}^{2} A(\mathbf{q})\right|^{2} \\
& -\frac{2 \pi^{2} K}{N} \sum_{\mathbf{q}} \frac{|m(\mathbf{q})|^{2}}{|Q|^{2}} \\
& \left.+\frac{2 \pi i}{N} \sum_{\mathbf{q}} \frac{Q_{x}}{|Q|^{2}} A(\mathbf{q}) m(-\mathbf{q})\right\} .
\end{aligned}
$$

Eq. 12 may be conveniently represented in real space in terms of $G_{0}(\mathbf{r})=\frac{1}{N} \sum_{\mathbf{q}} e^{-i \mathbf{q} \cdot \mathbf{r}} /|Q|^{2}$ and $G_{1}(\mathbf{r})=$ $\frac{1}{N} \sum_{\mathbf{q}} e^{-i \mathbf{q} \cdot \mathbf{r}} Q_{x} / Q_{y}|Q|^{2}$. For large $r$, it is useful to note that $G_{0}(\mathbf{r}) \sim \log r$ and $G_{1}(\mathbf{r}) \sim \arctan (y / x)$. We can now write

$$
\begin{aligned}
& \mathcal{Z}_{\mathrm{VM}} \\
& =\sum_{\{A\}} \sum_{\{m\}} \exp \left\{-2 \pi^{2} K \sum_{\mathbf{R}, \mathbf{R}^{\prime}} m(\mathbf{R}) G_{0}\left(\mathbf{R}-\mathbf{R}^{\prime}\right) m\left(\mathbf{R}^{\prime}\right)\right. \\
& -\frac{1}{2 K} \sum_{\mathbf{r}, \mathbf{r}^{\prime}}\left(\frac{\partial A}{\partial y}\right)_{\mathbf{r}} G_{0}\left(\mathbf{r}-\mathbf{r}^{\prime}\right)\left(\frac{\partial A}{\partial y}\right)_{\mathbf{r}^{\prime}} \\
& +2 \pi i \sum_{\mathbf{r}, \mathbf{R}^{\prime}}\left(\frac{\partial A}{\partial y}\right)_{\mathbf{r}} G_{1}\left(\mathbf{r}-\mathbf{r}^{\prime}\right) m\left(\mathbf{R}^{\prime}\right) \\
& \left.-\frac{1}{2 h} \sum_{\mathbf{r}}\left|\left(\frac{\partial A}{\partial y}\right)_{\mathbf{r}}\right|^{2}\right\} \text {. }
\end{aligned}
$$

The logarithmic interaction among the $m$ variables establishes the fact that they are the vortex degrees of freedom. In this representation it is appropriate to introduce the core energy for the vortices, after which the resulting model is identical to the one analyzed in Refs. 1 and 8 . The resulting system can be considered as a generalized Villain model, for which $\mathcal{Z}_{\mathrm{GVM}}=\sum_{\left\{m, m^{\prime}\right\}} e^{-\mathcal{H}_{\mathrm{GVM}}}$, with

$$
\begin{aligned}
\mathcal{H}_{\mathrm{GVM}} & =2 \pi^{2} K \sum_{\mathbf{R}, \mathbf{R}^{\prime}} m(\mathbf{R}) G_{0}\left(\mathbf{R}-\mathbf{R}^{\prime}\right) m\left(\mathbf{R}^{\prime}\right) \\
& +\frac{1}{2 K} \sum_{\mathbf{r}, \mathbf{r}^{\prime}} m^{\prime}(\mathbf{r}) G_{0}\left(\mathbf{r}-\mathbf{r}^{\prime}\right) m^{\prime}\left(\mathbf{r}^{\prime}\right) \\
& -2 \pi i \sum_{\mathbf{r}, \mathbf{R}^{\prime}} m^{\prime}(\mathbf{r}) G_{1}\left(\mathbf{r}-\mathbf{r}^{\prime}\right) m\left(\mathbf{R}^{\prime}\right) \\
& +\frac{1}{2 h} \sum_{\mathbf{r}} m^{\prime}(\mathbf{r})^{2}+E_{c} \sum_{\mathbf{R}} m(\mathbf{R})^{2}
\end{aligned}
$$

In Eq. 14 we have replaced $(\partial A / \partial y)_{\mathbf{r}} \rightarrow m^{\prime}(\mathbf{r})$ to make the duality of the model apparent: upon interchanging $E_{c} \leftrightarrow 1 / 2 h$ and changing $K \rightarrow 1 / 4 \pi^{2} K$, the partition sum is unchanged. It immediately follows that if there is an unbinding transition for the vortices (the $m$ variables), there must also be such a transition for the dislocations (the $m^{\prime}$ variables) at an appropriate location in the phase diagram. This duality was exploited in Ref. 8 to find the RG flow equations for large $E_{c}$ and small $h$. In the limit analyzed below, large $E_{c}$ and small $1 / 2 h$, we shall see that there is a dislocation unbinding transition. The duality immediately tells us there must also be vortex unbinding for small $E_{c}$ and large $1 / 2 h$. 
While $\mathcal{H}_{\text {GVM }}$ displays an elegant symmetry, it is hard to work with directly. The appearance of an imaginary term in the Hamiltonian makes the configurational weights in the partition sum complex and can lead to subtle complications, particularly if one wishes to replace the integer degrees of freedom with continuous ones, which we will need to do to perform an RG analysis. Furthermore, as discussed above the physics of vortex (dislocation) unbinding is profoundly affected by fluctuations of the strings (domain walls), which are now only implicit in Eq. 14. To see more clearly what is going on, it is worthwhile to return to a representation such as Eq. 9. We will proceed to do this, but in doing so we will also replace $\mathcal{H}_{\mathrm{GVM}}$ by a continuum model that is amenable to an RG analysis.

\section{Continuum Model}

To return from the vortex representation to the domain wall-dislocation representation, one only needs to apply the Poisson resummation formula to the $m$ sum in $\mathcal{Z}_{\mathrm{GVM}}$. However, for the RG analysis of this system we will want to develop an equivalent model that contains continuous rather than integer fields, and it is at this point that it is convenient to begin doing so. Whereas

$$
\begin{aligned}
& \mathcal{Z}_{\mathrm{GVM}} \\
= & \sum_{\{n\}} \sum_{\{A\}} \int \mathcal{D} \phi \exp \left\{-\mathcal{H}_{\mathrm{VM}}\left[\{n \rightarrow \phi\},\left\{m^{\prime} \rightarrow \frac{\partial A}{\partial y}\right\}\right]\right. \\
- & \left.E_{c} \sum_{\mathbf{R}} n^{2}(\mathbf{R})-2 \pi i \sum_{\mathbf{R}} \phi(\mathbf{R}) n(\mathbf{R})\right\}, \\
\equiv & \sum_{\{A\}} \int \mathcal{D} \phi \prod_{\mathbf{R}} \sum_{n(\mathbf{R})=-\infty}^{\infty} \exp \left\{-\mathcal{H}_{\mathrm{VM}}\left[\phi, \frac{\partial A}{\partial y}\right]\right. \\
- & \left.E_{c} \sum_{\mathbf{R}} n^{2}(\mathbf{R})-2 \pi i \sum_{\mathbf{R}} \phi(\mathbf{R}) n(\mathbf{R})\right\},
\end{aligned}
$$

is an exact representation of the generalized Villain model, we truncate the sums $\sum_{n(\mathbf{R})=-\infty}^{\infty}$ to $\sum_{n(\mathbf{R})=-1}^{1}$. For large $E_{c}$ this is an excellent approximation; vortices with large topological charge play little role in the properties of the system when the fugacity is small. Using $\sum_{n=-1}^{1} \exp \left(-2 \pi i n \phi-E_{c} \sum_{\mathbf{R}} n^{2}\right)=1+y \cos (2 \pi \phi) \approx$ $\exp [y \cos (2 \pi \phi)]$ with $y=2 e^{-E_{c}}$ twice the fugacity, we arrive at an intermediate model,

$$
\begin{aligned}
\mathcal{Z}^{\prime}=\sum_{\{A\}} \int \mathcal{D} \phi \exp \{ & -\mathcal{H}_{\mathrm{VM}}\left[\phi, \frac{\partial A}{\partial y}\right] \\
& \left.+y \sum_{\mathbf{R}} \cos (2 \pi \phi)\right\} .
\end{aligned}
$$

At this point we have one continuous and one integer field. To go over to a fully continuous model we make the replacement $\mathcal{Z}^{\prime} \rightarrow \mathcal{Z}=\int \mathcal{D} \phi \int \mathcal{D} a e^{-\mathcal{H}_{\text {eff }}[\phi, a]}$, with

$$
\begin{aligned}
\mathcal{H}_{\mathrm{eff}}=\int d^{2} r & {\left[\frac{1}{2 K}|\nabla \phi(\mathbf{r})+a(\mathbf{r}) \hat{\mathbf{x}}|^{2}+\frac{1}{2 h}\left(\frac{\partial a}{\partial y}\right)^{2}\right.} \\
& -y \cos (2 \pi \phi(\mathbf{r}))+y \\
& \left.-y_{a} \cos (2 \pi a(\mathbf{r}))+y_{a}\right]
\end{aligned}
$$

In Eq. 16, we have gone over from a lattice to a continuum representation, taking our lattice constant $a_{0} \equiv 1$ as our unit of length, and we have subtracted constants so that the ground state energy is zero. Although our replacement of the integer field $A$ with the continuous field $a$ is not a controlled approximation in the same sense as when we introduced $\phi$ in favor of $n$, we have constructed $\mathcal{H}_{\text {eff }}$ so that it has the same symmetry properties under translations of $a \rightarrow a+m$, with $m$ an integer, as had $\mathcal{H}_{\mathrm{GVM}}$, using the $y_{a}$ cosine term. Thus we expect $\mathcal{H}_{\text {eff }}$ and $\mathcal{H}_{\mathrm{GVM}}$ to have the same phases and types of transitions among them ${ }^{7}$. Our replacement of $\mathcal{H}_{\mathrm{GVM}}$ with $\mathcal{H}_{\text {eff }}$ is in fact no better or worse than our initial replacement of $\mathcal{H}_{X Y}$ with $\mathcal{H}_{\mathrm{VM}}$. $\mathcal{H}_{\mathrm{eff}}$ is the model that we will focus on in our RG analysis.

It is useful to recognize that the low-energy configurations of $(\phi, a)$ in $\mathcal{H}_{\text {eff }}$ mirror those of the Villain model (Eq. 5) with which we started. For example, if we consider configurations with $a=0$, then $\mathcal{H}_{\text {eff }}$ is a two dimensional sine-Gordon model, supporting kink excitations $^{11}$ that are directly analogous to domain walls. $\mathcal{H}_{\text {eff }}$ also supports solitons that represent screw dislocations. We can see this by considering configurations in which $\phi(x, y) \rightarrow 1$ as $x \rightarrow-\infty$ and $\phi(x, y) \rightarrow 0$ as $x \rightarrow \infty$. For $a=0$ this will force in a domain wall running along the $\hat{\mathbf{y}}$ direction. However, if we set $a(x, y)=\delta(x)$, then we can produce a configuration of vanishing energy and satisfy the boundary condition on $\phi$ with $\phi(x, y)=\Theta(-x)$, where $\Theta(-x)$ is a step function. A screw dislocation is forced into the system when we impose the boundary conditions $[\phi(x, y), a(x, y)] \rightarrow\left[\phi_{\mathrm{SGS}}(x), 0\right]$ as $y \rightarrow-\infty$, and $[\phi(x, y), a(x, y)] \rightarrow[\Theta(-x), \delta(x)]$ as $y \rightarrow \infty$, where $\phi_{\mathrm{SGS}}(x)$ is the kink soliton of the sine-Gordon model ${ }^{11}$. These boundary conditions guarantee that the domain wall must end somewhere in the bulk of the sample. This endpoint is the screw dislocation core. This is pictorially shown in Fig. 4.

Finally, while most parameters entering $\mathcal{H}_{\text {eff }}(K, h$, and $y)$ are directly related to parameters of the generalized Villain model $\left(K, h\right.$, and $\left.E_{c}\right)$, the value of $y_{a}$ in terms of these parameters is not immediately obvious. The connection can be made by recognizing that $y_{a}$ plays a key role in determining the dislocation core energy in $\mathcal{H}_{\text {eff }}$ : if $y_{a}$ is excessively large, then variations of $a$ in the $\hat{\mathbf{y}}$ direction become energetically very expensive, so that the core of a dislocation, where $a$ for example varies from 0 to $\delta(x)$, becomes high in energy.

Unfortunately the screw dislocation is a rather complicated soliton so that it is not obvious how one might obtain an analytic expression for its energy. We can, however, make a rough estimate for $y_{a}$ by looking at the dislocation configuration in the Hamiltonian implicit in 
(a) Closed wall configuration:

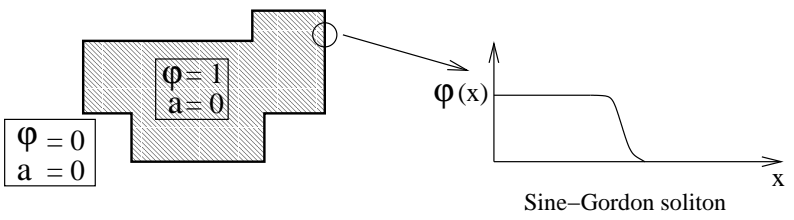

(b) Open wall configuration:

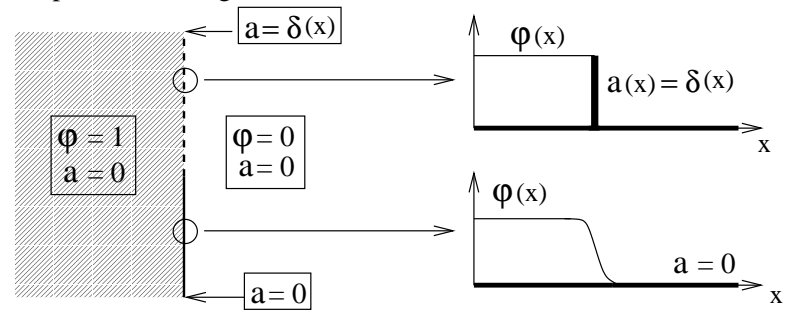

FIG. 4. Different low energy soliton configurations of $(\phi, a)$ for the effective Hamiltonian $\mathcal{H}_{\text {eff }}$ defined in Eq. 16. (a) For closed wall configurations with $a(x)=0, \mathcal{H}_{\mathrm{eff}}$ is a two-dimensional sine-Gordon equation with sine-Gordon solitons. (b) $a(x)=\delta(x)$ for large positive $y, a(x)=0$ for large negative $y$, and $\phi(x, y) \rightarrow 1$ as $x \rightarrow-\infty, \phi(x, y) \rightarrow 0$ as $x \rightarrow \infty$. The domain wall has finite energy per unit length as $y \rightarrow-\infty$, but vanishing energy per unit length as $y \rightarrow \infty$. The resulting configuration is a screw dislocation.

the partition sum for $\mathcal{Z}^{\prime}$ in Eq. 15. In Eq. 15, $A$ is an integer variable residing on the vertical bonds, and we create a dislocation near the origin by setting $A(x, Y)=-1$ for $x=0, Y>0$, and $A=0$ elsewhere, and introducing the same boundary conditions on $\phi$ described two paragraphs above. It is clear for large negative $Y$, $\phi$ will contain a sine-Gordon kink soliton, whereas for large positive $Y$, it has the form of a step function. The $\phi$ kink presumably narrows from the sine-Gordon width to zero in the vicinity of the origin. The energy of the configuration will take the form $\varepsilon=\varepsilon_{\phi}+1 / 2 h$, the first contribution representing the energy stored in the $\phi$ field and its interaction with $A$, and the second the energy cost for the step down in $A$ at the origin. In going over to $\mathcal{H}_{\text {eff }}$, where $A$ is replaced by the continuous field $a$, if we assume the contribution $\varepsilon_{\phi}$ is unchanged, then we can approximately match the dislocation core energies by matching the cost of the step down in $a$ with $1 / 2 h$. This is estimated by keeping only the second and fifth terms of the right hand side of Eq. 16, so that the step is again a sine-Gordon kink. The energy of the kink is ${ }^{11} 8 \sqrt{y_{a} / h}$; we obtain the match by setting $y_{a} \approx 1 / 256 h$.

With this estimate, it is possible to connect the phases of $\mathcal{H}_{\text {eff }}$ with those of the GVM and $X Y$ models. We now address this with an RG analysis.

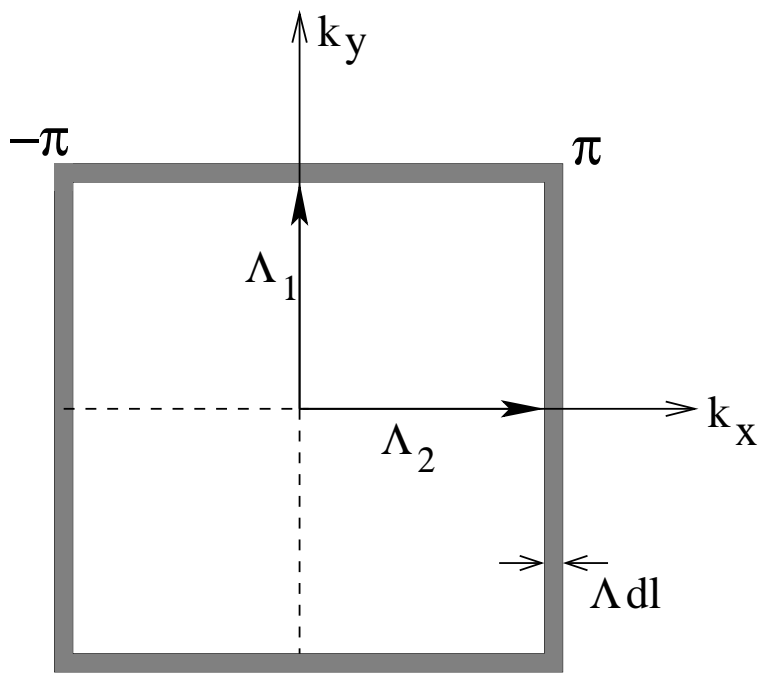

FIG. 5. Momentum shell in reciprocal space for the square lattice. $\boldsymbol{\Lambda}_{1}$ and $\boldsymbol{\Lambda}_{2}$ are equal length vectors of magnitude $\pi$ $\left(a_{0}=1\right)$ to the center of the BZ edges. The width of the shaded region is $\Lambda d \ell$.

\section{RENORMALIZATION GROUP ANALYSIS}

\section{A. Derivation of Scaling Relations}

Our analysis of $\mathcal{H}_{\text {eff }}$ proceeds with a momentum shell renormalization group procedure ${ }^{3}$ that is perturbative in $y$ and $y_{a}$. This involves dividing the fields into long and short wavelength components: $\phi(\mathbf{r})=\phi^{<}(\mathbf{r})+\phi^{>}(\mathbf{r})$, with

$$
\begin{aligned}
& \phi^{<}(\mathbf{r})=\int_{\left|q_{x}\right|,\left|q_{y}\right|<\Lambda / b} \frac{d^{2} \mathbf{q}}{(2 \pi)^{2}} e^{-i \mathbf{q} \cdot \mathbf{r}} \phi(\mathbf{q}), \\
& \phi^{>}(\mathbf{r})=\left(\int_{\Lambda / b<\left|q_{x}\right|<\Lambda}+\int_{\Lambda / b<\left|q_{y}\right|<\Lambda}\right) \frac{d^{2} \mathbf{q}}{(2 \pi)^{2}} e^{-i \mathbf{q} \cdot \mathbf{r}} \phi(\mathbf{q}) .
\end{aligned}
$$

There is an analogous decomposition for $a$. In Eqs. 17, $\Lambda=\pi / a_{0}, b=e^{\ell}$ is the rescaling factor, and we are including in $\phi^{>}$momentum components in a square shell at the edge of the Brillouin zone, as illustrated in Fig. 5.

As we shall see, the fixed points we are interested in are not orientationally isotropic, so it is important to choose the momentum shell so that it respects the symmetry of the lattice. (When we address the triangular lattice below, our momentum shell will be a hexagon.)

As usual ${ }^{7}$, the analysis proceeds by integrating out $\phi^{>}$ and $a^{>}$from the partition function $\mathcal{Z}$. For $y, y_{a}=0$ we then have

$$
\begin{aligned}
& \mathcal{Z}(\ell)=e^{-F(\ell)} \int \mathcal{D} \phi^{<} \int \mathcal{D} a^{<} \\
& \times \exp \left[-\frac{1}{2 K} \int^{<} \frac{d^{2} \mathbf{q}}{(2 \pi)^{2}}\left[\left|-i \mathbf{q} \phi^{<}(\mathbf{q})+a^{<}(\mathbf{q}) \hat{\mathbf{x}}\right|^{2}\right.\right. \\
&\left.\left.+\xi^{2}\left|a^{<}(\mathbf{q})\right|^{2}\right]\right],
\end{aligned}
$$


where $e^{-F(\ell)}$ is a prefactor that comes from the integrals over $\phi^{>}$and $a^{>}, \int^{<}$indicates an integral in the inner part of the Brillouin zone (i.e., inside the shaded region in Fig. 5) and $\xi=\sqrt{K / h}$ is the width of a string connecting a highly separated vortex-antivortex pair ${ }^{10}$. We can bring what remains of the Hamiltonian back to its original form (for $y, y_{a}=0$ ) with the scaling transformation

$$
\begin{gathered}
\mathbf{q}=\mathbf{q}^{\prime} / b, \quad \mathbf{r}=b \mathbf{r}^{\prime}, \\
\phi^{<}(\mathbf{r})=\phi^{\prime}\left(\mathbf{r}^{\prime}\right), a^{<}(\mathbf{r})=a^{\prime}\left(\mathbf{r}^{\prime}\right) / b .
\end{gathered}
$$

We then have in real space

$$
\begin{aligned}
& \mathcal{Z}(\ell)=e^{-F(\ell)} \quad \int \mathcal{D} \phi^{\prime} \int \mathcal{D} a^{\prime} \\
& \times \exp \left\{-\frac{1}{2 K^{\prime}} \int d^{2} r^{\prime}\left[\left|\nabla \phi^{\prime}\left(\mathbf{r}^{\prime}\right)+a^{\prime}\left(\mathbf{r}^{\prime}\right) \hat{\mathbf{x}}\right|^{2}\right.\right. \\
& \left.\left.+\xi^{\prime 2}\left|a^{\prime}\left(\mathbf{r}^{\prime}\right)\right|^{2}\right]^{2}\right\}
\end{aligned}
$$

with $K^{\prime}=K, \xi^{\prime 2}=\xi^{2} / b^{2}$. For a very narrow shell $(\ell \rightarrow d \ell, b \rightarrow 1+d \ell)$, the differential changes in $K$ and $\xi^{2}$ are just

$$
\begin{aligned}
& \frac{d K}{d \ell}=0, \\
& \frac{d \xi^{2}}{d \ell}=-2 \xi^{2} .
\end{aligned}
$$

Thus we have a set of fixed points whose stability with respect to the cosine perturbations we need to assess. For the $y \cos (2 \pi \phi)$ perturbation we will proceed in the standard fashion ${ }^{3,4}$, and we will see near the fixed points of interest it has a large anomalous dimension - the perturbation is highly irrelevant. The $y \cos (2 \pi a)$ perturbation, on the other hand, is rather unusual. The scaling relation for $a$ in Eq. 19 causes it to shrink as one integrates out the short wavelength fluctuations of the system. This suggests we should expand

$$
y_{a} \cos (2 \pi a)-y_{a}=\sum_{n=1}^{\infty} \frac{y_{2 n}}{(2 n) !}(-1)^{n}(2 \pi a)^{2 n},
$$

with the initial values $y_{2 n}(l=0)=y_{a}$. Our effective Hamiltonian is now

$$
\begin{aligned}
\mathcal{H}_{\mathrm{eff}}=\int d^{2} r & {\left[\frac{1}{2 K}|\nabla \phi(\mathbf{r})+a(\mathbf{r}) \hat{\mathbf{x}}|^{2}+\frac{1}{2 h}\left(\frac{\partial a}{\partial y}\right)^{2}\right.} \\
& -y \cos (2 \pi \phi(\mathbf{r}))+y \\
& \left.-\sum_{n=1}^{\infty} \frac{y_{2 n}}{(2 n) !}(-1)^{n}(2 \pi a)^{2 n}\right] .
\end{aligned}
$$

If we simply substitute $a(\mathbf{r}) \rightarrow a^{\prime}\left(\mathbf{r}^{\prime}\right) / b$ in the last term of Eq. 22, we see that the coefficients $y_{2 n} \sim b^{2-2 n}$. This suggests, as we will confirm below, that most of the terms in this expansion are irrelevant. However, the Gaussian (i.e., first) term is special because it neither grows nor shrinks, suggesting we should incorporate it into our fixed point. Writing $\rho(\ell) \equiv 4 \pi^{2} y_{2}(\ell)$, we take our unperturbed Hamiltonian to be

$$
\begin{aligned}
\mathcal{H}_{0}=\int d^{2} r & {\left[\frac{1}{2 K}|\nabla \phi(\mathbf{r})+a(\mathbf{r}) \hat{\mathbf{x}}|^{2}+\frac{1}{2} \rho a(\mathbf{r})^{2}\right.} \\
& \left.+\frac{\xi^{2}}{2 K}\left(\frac{\partial a}{\partial y}\right)^{2}\right] .
\end{aligned}
$$

To first order in $y, y_{a}$, the partition function takes the form

$$
\begin{aligned}
\mathcal{Z} & =\int \mathcal{D} \phi^{<} \int \mathcal{D} \phi^{>} \int \mathcal{D} a^{<} \int \mathcal{D} a^{>} e^{-\mathcal{H}_{0}} \\
& \times\left\{1-y \int d^{2} r \cos \left[2 \pi \phi^{<}(\mathbf{r})+2 \pi \phi^{>}(\mathbf{r})\right]\right. \\
& \left.-\int d^{2} r \sum_{n=2}^{\infty} \frac{y_{2 n}}{(2 n) !}(-1)^{n}\left[2 \pi a^{<}(\mathbf{r})+2 \pi a^{>}(\mathbf{r})\right]^{2 n}\right\} .
\end{aligned}
$$

The "1" term above has essentially been discussed already; the only difference one gets after integrating out $\phi^{>}, a^{>}$from Eq. 18 is that $F(\ell)$ now has a correction of order $y_{a}$ due to the $\rho$ term appearing in $\mathcal{H}_{0}$. Thus the scaling relations Eqs. 20 are correct to first order in $y$ and $y_{a}$. We next wish to integrate out the short wavelength fields in the middle term of Eq. 24. Calling this contribution $\mathcal{Z}_{y}$, the integral is

$$
\begin{aligned}
\mathcal{Z}_{y} & \equiv-y \int \mathcal{D} \phi^{<} \int \mathcal{D} \phi^{>} \int \mathcal{D} a^{<} \int \mathcal{D} a^{>} e^{-\mathcal{H}_{0}} \\
& \times\left\{\int d^{2} r \cos \left[2 \pi \phi^{<}(\mathbf{r})+2 \pi \phi^{>}(\mathbf{r})\right]\right\}, \\
& =-y e^{-F(\ell)} \\
& \times \exp \left\{-\frac{K}{2} \int^{>} d^{2} \mathbf{q}\left[q^{2}-\frac{q_{x}^{2}}{1+K \rho+\xi^{2} q_{y}^{2}}\right]^{-1}\right\} \\
& \times \int \mathcal{D} \phi^{<} \int \mathcal{D} a^{<} e^{-\mathcal{H}_{0}^{<}} \int d^{2} r \cos \left[2 \pi \phi^{<}(\mathbf{r})\right],
\end{aligned}
$$

where $\mathcal{H}_{0}^{<}$is obtained by Fourier transforming the right hand side of Eq. 23, and then dropping wavevectors in the resulting $\mathbf{q}$ integral that are in the momentum shell. We can already see the crucial role played by the parameter $\rho$ : for $\rho=0$, the $\int^{>} d^{2} \mathbf{q}$ integral is divergent, and it is apparent that $\mathcal{Z}_{y}$ vanishes in the thermodynamic limit. Since the values of $\rho$ in which we are interested are indeed small, it is apparent that the $y \cos (2 \pi \phi)$ is going to be strongly irrelevant. We estimate the $\int^{>} d^{2} \mathbf{q}$ integral by evaluating it for $\xi=0$ since this variable is irrelevant (Eq. 20). For small $\rho$, one finds

$$
\int^{>} d^{2} \mathbf{q}\left[q^{2}-\frac{q_{x}^{2}}{1+K \rho}\right]^{-1}=\pi K \sqrt{\frac{1+K \rho}{K \rho}} \log b+\mathcal{O}(\rho) .
$$

Rescaling the fields and lengths, we can return $\mathcal{Z}_{y}$ to its original form, writing

$$
\mathcal{Z}_{y}=-y^{\prime} e^{-F(\ell)} \int \mathcal{D} \phi \int \mathcal{D} a e^{-\mathcal{H}_{0}} \int d^{2} r \cos [2 \pi \phi(\mathbf{r})],
$$


with

$$
y^{\prime}=y b^{2-\pi K} \sqrt{\frac{1+K \rho}{K \rho}},
$$

where again we are focusing on small $\rho$. Recalling $b=$ $1+d \ell+\mathcal{O}\left(d \ell^{2}\right)$, we now have the scaling relation for $y$,

$$
\frac{d y}{d \ell}=\left[2-\pi K \sqrt{\frac{1+K \rho}{K \rho}}\right] y .
$$

Because $\rho$ is small (recall its initial value is $\mathcal{O}\left(y_{a}\right)$, and we shall see it shrinks under renormalization), for the parameters of interest $y$ shrinks very rapidly as we integrate out short wavelengths. The $y \cos (2 \pi \phi)$ term is thus strongly irrelevant, suggesting that the system is in a "rough" phase - domain walls are proliferated through the system. We will discuss the implications of this below.

We are left finally with deriving the scaling relations for the polynomial terms in $a$. These are also handled in a standard fashion ${ }^{3}$. We first consider a term in the last sum of Eq. 24. Expressed in terms of the Fourier components of the fields, such a term may be written as

$$
\begin{aligned}
\mathcal{Z}_{y_{2 n}} & \equiv-\int \mathcal{D} \phi^{<} \int \mathcal{D} \phi^{>} \int \mathcal{D} a^{<} \int \mathcal{D} a^{>} e^{-\mathcal{H}_{0}} \\
& \times \frac{y_{2 n}}{(2 n) !}(-1)^{n} \int d^{2} \mathbf{q}_{\mathbf{1}} d^{2} \mathbf{q}_{\mathbf{2}} \ldots d^{2} \mathbf{q}_{\mathbf{2 n}} \\
& \times(2 \pi)^{2} \delta\left(\mathbf{q}_{\mathbf{1}}+\mathbf{q}_{\mathbf{2}}+\ldots+\mathbf{q}_{\mathbf{2 n}}\right) \\
& \times a\left(\mathbf{q}_{\mathbf{1}}\right) a\left(\mathbf{q}_{\mathbf{2}}\right) \ldots a\left(\mathbf{q}_{\mathbf{2} \mathbf{n}}\right) .
\end{aligned}
$$

In writing this in terms of short and long-wavelength contributions, we wish to separate each of the $a\left(\mathbf{q}_{\mathbf{i}}\right)$ 's in the product above by the location of $\mathbf{q}_{\mathbf{i}}$ in the Brillouin zone. One thus has

$$
\begin{aligned}
& a\left(\mathbf{q}_{\mathbf{1}}\right) a\left(\mathbf{q}_{\mathbf{2}}\right) \ldots a\left(\mathbf{q}_{\mathbf{2} \mathbf{n}}\right) \rightarrow \\
& a^{<}\left(\mathbf{q}_{\mathbf{1}}\right) a^{<}\left(\mathbf{q}_{\mathbf{2}}\right) \ldots a^{<}\left(\mathbf{q}_{\mathbf{2 n}}\right) \\
+ & \frac{2 n(2 n-1)}{2} a^{>}\left(\mathbf{q}_{\mathbf{1}}\right) a^{>}\left(\mathbf{q}_{\mathbf{2}}\right) a^{<}\left(\mathbf{q}_{\mathbf{3}}\right) \ldots a^{<}\left(\mathbf{q}_{\mathbf{2} \mathbf{n}}\right)+\ldots,
\end{aligned}
$$

where we have dropped the term with just one $a^{>}$, anticipating this will integrate to zero in the functional integral. Because our momentum shell has width $d \ell$, we need only retain the two terms explicitly shown in Eq. 28; terms with more factors of $a^{>}$involve higher powers of $d \ell$ and vanish when we take $d \ell \rightarrow 0$. Substituting this expansion into Eq. 27, we obtain

$$
\begin{aligned}
\mathcal{Z}_{y_{2 n}} & =-y_{2 n} \int \mathcal{D} \phi^{<} \int \mathcal{D} a^{<} e^{-\mathcal{H}_{0}^{<}} \\
& \times e^{-F(\ell)} \int d^{2} r\left\{\frac{(-1)^{n}}{(2 n) !}\left[2 \pi a^{<}(\mathbf{r})\right]^{2 n}\right. \\
& \left.-\frac{(-1)^{n-1}}{(2 n-2) !} 2 \pi^{2} \mathcal{L}(\rho, \xi) d \ell\left[2 \pi a^{<}(\mathbf{r})\right]^{2 n-2}\right\},
\end{aligned}
$$

with

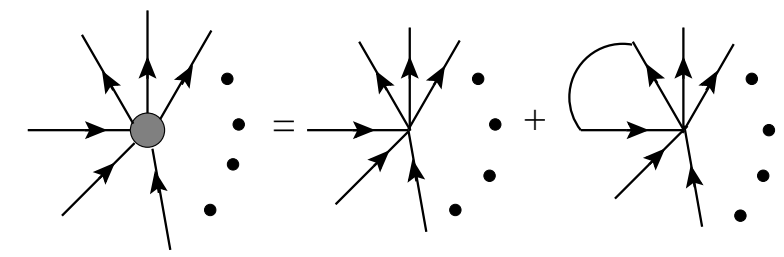

FIG. 6. Pictorial representation of Eq. 29. Integral over short wavelength fields $\left[\phi^{>}(\mathbf{r})\right]$ of the $y_{2 n}$ term introduces a renormalization of the $y_{2 n-2}$ term. The graphical decomposition of a $2 n$ point vertex is shown. The first diagram on the right hand side is a tree diagram that represents all the long-wavelength fields $\left[\phi^{<}(\mathbf{r})\right]$ which correspond to the first term in Eq. 29. Contraction between a pair of legs is shown in the second diagram and it represents the second term in Eq. 29. The black dots in the figure represent other legs.

$$
\begin{aligned}
e^{-F(\ell)} \mathcal{L}(\rho, \xi) d \ell & =\int \mathcal{D} \phi^{>} \int \mathcal{D} a^{>} e^{-\mathcal{H}_{0}^{>}} \\
& \times \int^{>} \frac{d^{2} \mathbf{q}}{(2 \pi)^{2}} a^{>}(-\mathbf{q}) a^{>}(\mathbf{q}) .
\end{aligned}
$$

Eq. 29 shows that the integral over short wavelengths of the $y_{2 n}$ term introduces a renormalization of the $y_{2 n-2}$ term. This is illustrated graphically in Fig. 6. Rescaling fields and lengths, we see that $\mathcal{Z}_{y_{2 n}}$ may also be returned to its original form,

$$
\mathcal{Z}_{y_{2 n}}=-y_{2 n}^{\prime} e^{-F(\ell)} \int \mathcal{D} \phi \int \mathcal{D} a e^{-\mathcal{H}_{0}}\left\{\frac{(-1)^{n}}{(2 n) !}[2 \pi a(\mathbf{r})]^{2 n}\right\}
$$

with

$$
y_{2 n}^{\prime}=y_{2 n} b^{2-2 n}-2 \pi^{2}[\mathcal{L}(\rho, \xi) d \ell] y_{2 n+2} .
$$

This allows us to write down our last scaling relation,

$$
\frac{d y_{2 n}}{d l}=-(2 n-2) y_{2 n}-2 \pi^{2} \mathcal{L}(\rho, \xi) y_{2 n+2} .
$$

A slightly subtle but important question arises when we recall $4 \pi^{2} y_{2}=\rho$. Should Eq. 32 be applied to $\rho$, even though it appears in $\mathcal{H}_{0}$ and is not included in the $\mathcal{Z}_{y_{2 n}}$ 's? The answer is yes; the integration of the $a^{4}$ term does produce a Gaussian term that renormalizes the $\frac{1}{2} \rho a^{2}$ in $\mathcal{H}_{0}$. This is easily seen if we reexponentiate the $\mathcal{O}\left(y_{a}\right)$ contribution to the partition function. The renormalizations of the $y_{2 n}$ terms follow through exactly as we have described above, and one can then see explicitly that the $y_{4}$ term renormalizes $\rho$. Thus we can write for the $n=1$ case of Eq. 32

$$
\frac{d \rho}{d l}=-8 \pi^{4} \mathcal{L}(\rho, \xi) y_{4} .
$$

The derivation of the scaling relations is completed by writing down an explicit expression for $\mathcal{L}(\rho, \xi)$. The functional integrals in Eq. 30 yield 


$$
\mathcal{L}(\rho, \xi) d \ell=\int^{>} \frac{d^{2} \mathbf{q}}{(2 \pi)^{2}} \frac{2 K q^{2}}{q_{y}^{2}+\left[K \rho+\xi^{2} q_{y}^{2}\right] q^{2}} .
$$

This integral can be computed exactly in a tedious but in principle straightforward calculation. The result is displayed in Appendix A. For small $\rho$, the result (Eq. 60) may be expanded to yield

$$
\mathcal{L} \approx \frac{K \Lambda^{2}}{\pi \sqrt{K \rho\left(1+\xi^{2} \Lambda^{2}\right)}} .
$$

Eq. 35 once again demonstrates the importance of the parameter $\rho$ : as $\rho \rightarrow 0, \mathcal{L}$ diverges, suggesting that the $\frac{1}{2} \rho a^{2}$ term in $\mathcal{H}_{0}$ is irrelevant if the initial value of $\rho$ is small enough. We will see this is indeed the case.

Eqs. 20, 26, 32, and 33 are the scaling equations for our problem. Before presenting results obtained from numerically integrating them in Sec. V, we discuss the fixed points of the equations and their interpretation.

\section{B. Fixed Points: Bound and Unbound Dislocation States}

It is easy to see that fixed points of Eqs. 20, 26, 32, and 33 occur for $y=0, y_{2 n \geq 4}=0$, and $\xi^{2}=0$, so that the generic fixed point Hamiltonian has the form

$$
H_{*}=\int d^{2} r\left[\frac{1}{2 K}|\nabla \phi(\mathbf{r})+a(\mathbf{r}) \hat{\mathbf{x}}|^{2}+\frac{1}{2} \rho a(\mathbf{r})^{2}\right] .
$$

We will see in our numerical integration of the scaling relations that any (small) value of $\rho$ is an attractive fixed point. This will give us an unbroken line of fixed points. In spite of the $\rho=0$ point being connected to the $\rho>0$ fixed points, these two cases are physically different. The reason goes back to the role $a$ plays in allowing us to form separated dislocation pairs. We demonstrated in the last section that one can remove the energy of a section of domain wall in the $\phi$ field when $\rho=0$, by creating a narrow line segment where $a \neq 0$ along some part of the domain wall (see Fig. 4). Because the $y \cos (2 \pi \phi)$ term is irrelevant, we understand that domain walls in $\phi$ proliferate through the system. Thus, if we consider only $a=0$ configurations, we can find arbitrarily large domain wall loops. Such loops can be broken open to create dislocation pairs. The only extra energy cost for doing this to a closed loop of length $L$ is twice the dislocation core energy; the loop can be deformed to move the dislocations a distance $\sim L$ apart, without any further cost in energy. This finite core energy is more than balanced by the entropy associated with the number of open domain wall configurations for large $L$, so that large open domain wall configurations - highly separated dislocation states - are not suppressed by the dislocation core energy. We thus identify the $\rho=0$ fixed point as an unbound dislocation state.

For any non-vanishing value of $\rho$, the situation for large enough $L$ becomes profoundly different. The "mass" term for $a$ tells us that we cannot completely eliminate the domain wall energy. If we create a dislocation pair by deforming a closed loop, there is a residual attractive interaction between them. We can thus identify $\rho$ as a renormalized string tension for the dislocation pair. When $\rho>0$, the dislocations are bound into pairs. It is interesting to note that because the $a$ field lives only on the vertical bonds of the underlying lattice, we do not get the configurational entropy that the domain walls in the $\phi$ field has - so even though the residual "string" connecting the dislocations may have much lower energy per unit length than the closed domain wall loops, the free energy of closed domain walls can vanish, while that of the residual strings remains finite.

While it is tempting to interpret the energy of the residual strings as leading to linear confinement, this is not correct because it ignores the effect of fluctuations in $\phi$. A simple interpretation may be had if we interpret $\rho>0$ as telling us that the "integerness" of the original $A$ field cannot be ignored - the $\cos (2 \pi a)$ term contains physics which is relevant at long wavelengths - even while the irrelevance of $y$ tells us we may treat $\phi$ as a continuous field and ignore the $\cos (2 \pi \phi)$ perturbation. This suggests we can return to $\mathcal{Z}^{\prime}$ (Eq. 15), setting $y=0$, which allows us to integrate out $\phi$. Up to unimportant prefactors, one finds $\mathcal{Z}^{\prime} \propto \sum_{\{A\}} e^{-\mathcal{H}_{C G}}$, with

$$
\begin{aligned}
\mathcal{H}_{C G} & =\frac{1}{2 K} \sum_{\mathbf{r}, \mathbf{r}^{\prime}}\left(\frac{\partial A}{\partial y}\right)_{\mathbf{r}} G_{0}\left(\mathbf{r}-\mathbf{r}^{\prime}\right)\left(\frac{\partial A}{\partial y}\right)_{\mathbf{r}^{\prime}} \\
& \left.+\frac{1}{2 h} \sum_{\mathbf{r}}\left|\left(\frac{\partial A}{\partial y}\right)_{\mathbf{r}}\right|^{2}\right\} .
\end{aligned}
$$

Recalling that $(\partial A / \partial y)$ is a representation of the dislocation field, we see that we have come to a Coulomb gas Hamiltonian! This suggests we should think of the $\rho>0$ state as a set of logarithmically interacting dislocations, rather than linearly confined ones. However, in coming to $H_{C G}$ we understood $\rho>0$ as signaling that we cannot ignore the discreteness of the underlying charge. This is the usual situation when the charges are in a bound state. If one can treat $(\partial A / \partial y) \rightarrow(\partial a / \partial y)$ as a continuous field, the state may be interpreted as a "charged liquid" ${ }^{21}$ and the dislocations may be treated as unbound. This is only valid if $\rho=0$.

An alternative look at the fixed points may be had by reexpressing them in terms of the vortices. To do this, we return $\phi$ to its integer form $n$ but keep $a$ as a continuous field, adopting the fixed point form for its energetics. Applying the Poisson resummation formula to $n$ brings us to an effective partition function $\tilde{\mathcal{Z}}^{\prime}=$ $\sum_{\{m\}} \int \mathcal{D} a e^{-\tilde{\mathcal{H}}^{\prime}}$ with

$$
\begin{aligned}
\tilde{\mathcal{H}}^{\prime}=\frac{1}{N} \sum_{\mathbf{q}} & \left\{\left[\frac{1}{2 K|Q|^{2}}\right]\left|Q_{y}^{2} a(\mathbf{q})\right|^{2}+\frac{\rho}{2}|a(\mathbf{q})|^{2}\right. \\
+ & \left.2 \pi^{2} K \frac{|m(\mathbf{q})|^{2}}{|Q|^{2}}-2 \pi i \frac{Q_{x}}{|Q|^{2}} a(\mathbf{q}) m(-\mathbf{q})\right\} .
\end{aligned}
$$


Integrating out $a$ gives, again up to unimportant prefactors, $\tilde{\mathcal{Z}}^{\prime}=\sum_{\{m\}} e^{-\tilde{\mathcal{H}}}$, with the reduced Hamiltonian $\tilde{\mathcal{H}}$ now

$$
\tilde{\mathcal{H}}=\frac{2 \pi^{2} K}{N} \sum_{\mathbf{q}} \frac{|m(\mathbf{q})|^{2}}{|Q|^{2}}\left[1+\frac{\left|Q_{x}\right|^{2}}{\left|Q_{y}\right|^{2}+\rho K|Q|^{2}}\right] .
$$

The energetics of the vortices is profoundly different for $\rho>0$ and $\rho=0$. This is most clearly seen by considering a configuration for a single vortex at the origin, $m(\mathbf{R})=$ $\delta_{\mathbf{R}, 0}$, in a finite size system of linear dimension $L$. The energy of such a configuration has the form

$$
\begin{aligned}
<\tilde{\mathcal{H}}>_{1 v} & \sim(1+C) \log L, \quad(\rho>0) \\
& \sim L, \quad(\rho=0)
\end{aligned}
$$

where $C=\int_{0}^{2 \pi} d \theta\left[\cos ^{2} \theta /\left(\sin ^{2} \theta+\rho K\right)\right]$. We find the usual logarithmic energy for a vortex when $\rho>0$, although the correction $C$ to the coupling constant becomes very large for small $\rho$. The effect of the dislocations thus is a strong upward renormalization of the effective $K$ in this state. For $\rho=0$, by contrast, the energy of a single vortex configuration grows linearly with $L$. Thus, the state that we identified as the unbound dislocation state becomes, in the dual representation, a linearly confined vortex state. By contrast, the $\rho>0$ state represents logarithmically confined vortices, or, in the dual language, logarithmically bound dislocations. Apparently these dual defects are in the same state at such fixed points.

The duality of the partition function tells us then that there are three possible states for the vortices, linearly confined, logarithmically bound, or deconfined the last because the symmetry of the effective Hamiltonian $\mathcal{H}_{G V M}$ (Eq. 14) tells us that if a deconfined dislocation state exists, so must a deconfined vortex state. Recent simulation studies ${ }^{14}$ have strongly supported the existence of three such states in the $X Y$ model. For example, if one fixes $K$ below the critical value for the Kosterlitz-Thouless transition, then at small $h$ one finds fluctuations that scale with system size $L$ in a way expected for unbound vortices. At intermediate values of $h$, the fluctuations behave as if the vortices are in pairs that are interacting logarithmically at large separations. For larger values of $h$, they behave like dipoles with linear confinement ${ }^{14}$.

Finally, one may notice that $\tilde{\mathcal{H}}$ is not invariant under $q_{x} \leftrightarrow q_{y}$, so that the fixed points we are examining do not retain the full symmetry of the underlying lattice. Indeed, the loss of symmetry was been apparent in our development of the RG scaling relations, for which we saw $\mathcal{H}_{0}$ and ultimately $\mathcal{H}_{*}$ has configurations of vanishing energy along the $q_{y}=0$ axis, but not on the $q_{x}=0$ axis. This problem originated when we went from $\mathcal{H}_{\mathrm{GVM}}$ to our $\mathcal{H}_{\text {eff }}$. For the former model, the energetics of a dislocation with a domain wall exiting along a vertical bond is the same for one in which it exits along a horizontal bond; for the latter, they are different. The model can be formulated in a way that retains the lattice symmetry, at the sacrifice of some of the simplicity of the model we are currently analyzing. We will detail how this is done below. For now we note these models generate unbound dislocation (and vortex) fixed points that have not one but several nodal lines in the Brillouin zone, in a way that respects the orientational symmetry of the lattice so that the $\mathcal{H}_{*}$ we are currently analyzing is really only one of a class of fixed point Hamiltonians that can arise in $X Y$ spin models with symmetry-breaking fields.

\section{Integration of Scaling Relations}

We now need to show that there are parameter regimes for which $\mathcal{H}_{\text {eff }}$ flows to $\mathcal{H}_{*}$ both with $\rho=0$ and $\rho>$ 0 . The first two scaling relations - Eqs. 20 - are trivial to deal with. The first of these simply states that $K$ is invariant as we integrate out short wavelengths: we simply take this to be a constant in the discussion that follows. The second is easily integrated to give

$$
\xi^{2}(\ell)=\xi_{0}^{2} e^{-2 \ell},
$$

where $\xi_{0}=\sqrt{K / h}$ is the bare string width. Because of the simple relation between $\xi$ and $\ell$, it is convenient to characterize the length scale to which we have integrated in terms of $\xi$; i.e., $b \equiv e^{\ell}=\xi_{0} / \xi(\ell)$. Thus we will present RG flows below in terms of $\xi$.

Eq. 26 is also easy to deal with since the parameter $y$ does not renormalize any of the $y_{2 n}$ 's. Since the initial value of $\rho$ is small in our perturbative approach, and it shrinks under the RG, $y(\ell)$ shrinks rapidly as $\xi(\ell) \rightarrow 0$. We will not show this explicitly.

Eqs. 32 by contrast appear formidable since they present an infinite tower of equations. However, because the shell integral $\mathcal{L}$ is the same for each of these, the solutions of the equations are related to one another by $y_{2 n+2}=e^{-2 \ell} y_{2 n} \equiv \xi^{2} y_{2 n} / \xi_{0}^{2}$, as may easily be confirmed by direct substitution. Recalling $\rho=4 \pi^{2} y_{2}$, this tells us Eq. 33 may be written as

$$
\frac{d \rho}{d \ell}=-\frac{2 \pi^{2} \mathcal{L} \rho \xi^{2}}{\xi_{0}^{2}}
$$

Finally, noting $d \xi^{2} / d \ell=-2 \xi_{0}^{2} e^{-2 \ell}=-2 \xi^{2}(\ell)$, we can rewrite this as

$$
\frac{d \rho}{d \xi^{2}}=\frac{\pi^{2} \mathcal{L}(\rho, \xi) \rho}{\xi_{0}^{2}}
$$

Eq. 39 is particularly convenient for integration. Note the explicit dependence on $\xi_{0}^{2}$ shows that the renormalization of $\rho$ is strongest for the largest values of $h$ : $\rho$ scales to zero for large values of $h$, leading to the unconfined dislocation phase.

Fig. 7 shows a typical result for the integration of Eq. 39. For these flows, we have used our estimate of $y_{a}$ in terms of $h$ to fix the initial value of $\rho$, so that all the flows begin from the same point in the plot. The 


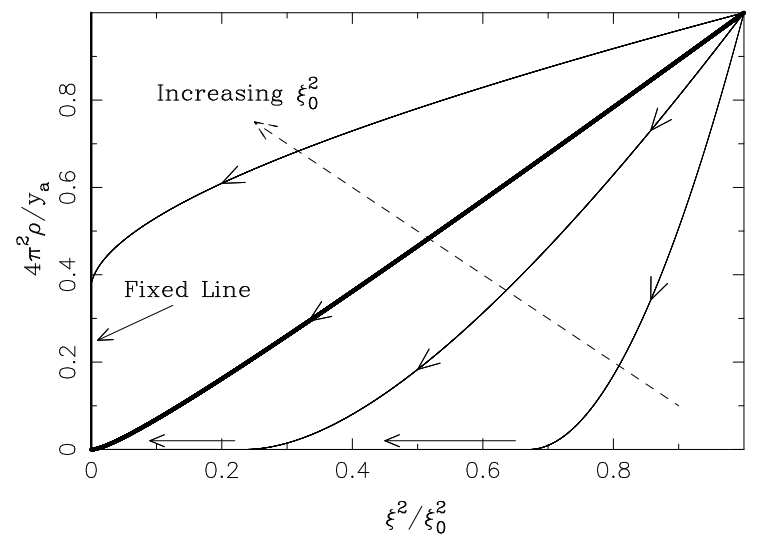

FIG. 7. RG flows for Eq. 39. Left vertical axis is a fixed line, and heavy line is a separatrix between flows that reach $\rho=0$ for finite $l$ and flow to the origin as $l \rightarrow \infty$, and those that have $\rho>0$ at the end of their flow. Finite values of $\rho$ in $H_{*}$ indicate dislocation binding.

structure that was described in the Introduction is apparent: there is a fixed line of $\rho$ values, with the $\rho=0$ point - the unbound dislocation phase - an endpoint of this line. For small values of $\xi_{0}, \rho(\ell)$ lies below the separatrix (shown as a heavy line), touches the $\rho=0$ axis at a finite value of $\xi^{2} / \xi_{0}^{2} \equiv e^{-2 \ell^{*}}$, and remains on this axis as it flows to the origin. Note that the divergence in $\mathcal{L}$ as $\rho \rightarrow 0$ guarantees the flows never cross the $\rho=0$ plane to negative values, which would indicate an instability in our model. We can interpret $\ell_{s c r}=a_{0} e^{\ell^{*}}$ as a screening length: for separations below this the dislocations appear to be bound, while for larger separations the interaction is screened by other dislocations, allowing for an unbinding transition. Apparently $\ell_{s c r}$ diverges as the transition is approached. This means the deconfinement transition has a feature in common with other more standard phase transitions: there is a diverging length scale. Above the separatrix, the flows end at a non-vanishing value of $\rho$, which as discussed above indicates the dislocations are in a bound state. The separatrix represents a deconfinement line, with dislocations unbound if $\xi_{0}$ is smaller than some critical value $\xi_{c r}$ for a fixed value of $K$.

The numerical integration of the scaling relations allow us to find some critical exponents for how $\rho$ develops and how $\ell_{s c r}$ diverges as the phase boundary is approached. Before giving results for these, we will demonstrate that the lattice symmetry can be properly incorporated into the model and find the scaling relations analogous to those above. This is the subject of the next section.

\section{FORMULATION OF THE MODEL WITH FULL LATTICE SYMMETRY}

\section{A. Square Lattice}

In this section we will formulate the theory retaining the full underlying symmetry of the square lattice. We begin by defining the bond variables $S_{1}$ and $S_{2}$ in terms of integer fields $m_{1}$ and $m_{2}$ on the dual lattice sites $\{\mathbf{R}\}$ as in Eq. 7 and substitute into Eq. 5 to obtain in momentum space

$$
\begin{aligned}
\mathcal{H}_{\mathrm{VM}} & =\frac{1}{N} \sum_{\mathbf{q}} \frac{1}{2 K}\left[\left|Q_{1} m_{1}(\mathbf{q})\right|^{2}+\left|Q_{2} m_{2}(\mathbf{q})\right|^{2}\right] \\
& +\frac{1}{N} \sum_{\mathbf{q}} \frac{1}{2 h}\left|Q_{1} Q_{2} m_{1}(\mathbf{q})+Q_{1} Q_{2} m_{2}(\mathbf{q})\right|^{2},
\end{aligned}
$$

where $Q_{1}=1-e^{-i \mathbf{q} \cdot \boldsymbol{\Delta}_{1}}$ and $Q_{2}=1-e^{-i \mathbf{q} \cdot \boldsymbol{\Delta}_{\mathbf{2}}}$. As previously, to carry out an RG analysis we need to replace $m_{1,2}$ by continuous fields $\phi_{1,2}$ and add in cosine terms which tend to preserve the "integerness" of the fields, as well their sums and differences. Most such terms work out to be irrelevant; however, one particular combination turns out to be analog of the $y_{a} \cos (2 \pi a)$ term of the last section. This is

$$
\begin{aligned}
\mathcal{H}^{\prime} & =-y_{a} \sum_{\mathbf{r}}\left[\left(1-\cos \left\{2 \pi\left[\phi_{1}\left(\mathbf{r}+\boldsymbol{\Delta}_{\mathbf{2}}\right)-\phi_{1}(\mathbf{r})\right]\right\}\right)\right. \\
& \left.+\left(1-\cos \left\{2 \pi\left[\phi_{2}\left(\mathbf{r}+\boldsymbol{\Delta}_{\mathbf{1}}\right)-\phi_{2}(\mathbf{r})\right]\right\}\right)\right] .
\end{aligned}
$$

It is useful now to do a Fourier transform of the integer fields $m_{1}$ and $m_{2}$ in Eq. 41 and expand the result in the small q limit

$$
\begin{aligned}
& \phi_{1}\left(\mathbf{r}+\boldsymbol{\Delta}_{\mathbf{2}}\right)-\phi_{1}(\mathbf{r}) \approx \frac{1}{N} \sum_{\mathbf{q}}\left(i \mathbf{q} \cdot \boldsymbol{\Delta}_{\mathbf{2}}\right) e^{i \mathbf{q} \cdot \mathbf{r}} \phi_{1}(\mathbf{q}), \\
& \phi_{2}\left(\mathbf{r}+\boldsymbol{\Delta}_{\mathbf{1}}\right)-\phi_{2}(\mathbf{r}) \approx \frac{1}{N} \sum_{\mathbf{q}}\left(i \mathbf{q} \cdot \boldsymbol{\Delta}_{\mathbf{1}}\right) e^{i \mathbf{q} \cdot \mathbf{r}} \phi_{2}(\mathbf{q}) .
\end{aligned}
$$

The effective Hamiltonian is now

$$
\begin{aligned}
\mathcal{H}_{\mathrm{eff}} & =\frac{1}{N} \sum_{\mathbf{q}}\left\{\frac{1}{2 K}\left[\left(\mathbf{q} \cdot \boldsymbol{\Delta}_{\mathbf{1}}\right)^{2}\left|\phi_{1}(\mathbf{q})\right|^{2}+\left(\mathbf{q} \cdot \boldsymbol{\Delta}_{\mathbf{2}}\right)^{2}\left|\phi_{2}(\mathbf{q})\right|^{2}\right]\right. \\
& +\frac{1}{2 h} \mid\left(\mathbf{q} \cdot \boldsymbol{\Delta}_{\mathbf{1}}\right)\left(\mathbf{q} \cdot \boldsymbol{\Delta}_{\mathbf{2}}\right) \phi_{1}(\mathbf{q}) \\
& \left.+\left.\left(\mathbf{q} \cdot \boldsymbol{\Delta}_{\mathbf{2}}\right)\left(\mathbf{q} \cdot \boldsymbol{\Delta}_{\mathbf{1}}\right) \phi_{2}(\mathbf{q})\right|^{2}\right\} \\
& -y_{a} \sum_{\mathbf{r}}\left\{\left(1-\cos \left[\frac{2 \pi}{N} \sum_{\mathbf{q}}\left(i \mathbf{q} \cdot \boldsymbol{\Delta}_{\mathbf{2}}\right) e^{i \mathbf{q} \cdot \mathbf{r}} \phi_{1}(\mathbf{q})\right]\right)\right. \\
& \left.+\left(1-\cos \left[\frac{2 \pi}{N} \sum_{\mathbf{q}}\left(i \mathbf{q} \cdot \boldsymbol{\Delta}_{\mathbf{1}}\right) e^{i \mathbf{q} \cdot \mathbf{r}} \phi_{2}(\mathbf{q})\right]\right)\right\} \\
& +y \sum_{\mathbf{r}}\left\{\left(1-\cos \left[2 \pi \phi_{1}(\mathbf{r})\right]\right)\right.
\end{aligned}
$$




$$
\left.+\left(1-\cos \left[2 \pi \phi_{2}(\mathbf{r})\right]\right)\right\} .
$$

In coming to Eq. 42, we have taken the long wavelength limit $Q_{1}=i \mathbf{q} \cdot \boldsymbol{\Delta}_{\mathbf{1}}$ and $Q_{2}=i \mathbf{q} \cdot \boldsymbol{\Delta}_{\mathbf{2}}$. We note the symmetry of $\mathcal{H}_{\text {eff }}$ in Eq. 42 under $\hat{\mathbf{x}} \leftrightarrow \hat{\mathbf{y}}$ and $\phi_{1} \leftrightarrow \phi_{2}$ preserves the square symmetry.

We are now set to perform the momentum shell renormalization group procedure on the above effective Hamiltonian that is perturbative in $y_{a}$. Note that the $y$ term is irrelevant and shrinks to zero as discussed in Sec. III C. Our analysis that follows is similar to that described in Section III - we divide the fields $\phi_{i}$ into long and short wavelength components $\phi_{i}^{>}$and $\phi_{i}^{<}$, integrate out the short wavelength fields (within the momentum shell as shown in Fig. 5) which give an overall prefactor in the partition function, and finally rescale the fields as in Eq. 19. In doing this we expand the $y_{a}$ cosine terms in Eq. 41 and incorporate their Gaussian contribution into our unperturbed Hamiltonian, which now becomes

$$
\begin{aligned}
\mathcal{H}_{0}=\frac{1}{N} \sum_{\mathbf{q}} & {\left[\frac{1}{2 K}\left\{\left(\mathbf{q} \cdot \boldsymbol{\Delta}_{\mathbf{1}}\right)^{2}+\rho K\left(\mathbf{q} \cdot \boldsymbol{\Delta}_{\mathbf{2}}\right)^{2}\right\}\left|\phi_{1}(\mathbf{q})\right|^{2}\right.} \\
& +\frac{1}{2 K}\left\{\left(\mathbf{q} \cdot \boldsymbol{\Delta}_{\mathbf{2}}\right)^{2}+\rho K\left(\mathbf{q} \cdot \boldsymbol{\Delta}_{\mathbf{1}}\right)^{2}\right\}\left|\phi_{2}(\mathbf{q})\right|^{2} \\
& +\frac{\xi^{2}}{2 K} \mid\left(\mathbf{q} \cdot \boldsymbol{\Delta}_{\mathbf{1}}\right)\left(\mathbf{q} \cdot \boldsymbol{\Delta}_{\mathbf{2}}\right) \phi_{1}(\mathbf{q}) \\
& \left.+\left.\left(\mathbf{q} \cdot \boldsymbol{\Delta}_{\mathbf{2}}\right)\left(\mathbf{q} \cdot \boldsymbol{\Delta}_{\mathbf{1}}\right) \phi_{2}(\mathbf{q})\right|^{2}\right],
\end{aligned}
$$

where $\rho(\ell)=4 \pi^{2} y_{2}(\ell)$ and $\xi(\ell=0)=\sqrt{K / h}$. After rearrangement, the resulting unperturbed Hamiltonian takes the form,

$$
\begin{aligned}
\mathcal{H}_{0}=\frac{1}{N} \sum_{\mathbf{q}} & {\left[\frac { 1 } { 2 K } \left\{\left(\mathbf{q} \cdot \boldsymbol{\Delta}_{\mathbf{1}}\right)^{2}+\rho K\left(\mathbf{q} \cdot \boldsymbol{\Delta}_{\mathbf{2}}\right)^{2}\right.\right.} \\
& \left.+\xi^{2}\left(\mathbf{q} \cdot \boldsymbol{\Delta}_{\mathbf{1}}\right)^{2}\left(\mathbf{q} \cdot \boldsymbol{\Delta}_{\mathbf{2}}\right)^{2}\right\}\left|\phi_{1}(\mathbf{q})\right|^{2} \\
& +\frac{1}{2 K}\left\{\left(\mathbf{q} \cdot \boldsymbol{\Delta}_{\mathbf{2}}\right)^{2}+\rho K\left(\mathbf{q} \cdot \boldsymbol{\Delta}_{\mathbf{1}}\right)^{2}\right. \\
& \left.+\xi^{2}\left(\mathbf{q} \cdot \boldsymbol{\Delta}_{\mathbf{2}}\right)^{2}\left(\mathbf{q} \cdot \boldsymbol{\Delta}_{\mathbf{1}}\right)^{2}\right\}\left|\phi_{2}(\mathbf{q})\right|^{2} \\
& \left.+\frac{\xi^{2}}{K}\left(\mathbf{q} \cdot \boldsymbol{\Delta}_{\mathbf{1}}\right)^{2}\left(\mathbf{q} \cdot \boldsymbol{\Delta}_{\mathbf{2}}\right)^{2} \phi_{1}(\mathbf{q}) \phi_{2}(\mathbf{q})\right] .
\end{aligned}
$$

As in Sec. III A, the fixed points of the RG equations [Eqs. 20, 26, 32, and 33] occur for $y_{2 n \geq 4}=0$, and $\xi^{2}=0$, so that the generic fixed point Hamiltonian is

$$
\begin{aligned}
\mathcal{H}_{*} & =\frac{1}{N} \sum_{\mathbf{q}}\left[\frac{1}{2 K}\left\{\left(\mathbf{q} \cdot \boldsymbol{\Delta}_{\mathbf{1}}\right)^{2}+\rho K\left(\mathbf{q} \cdot \boldsymbol{\Delta}_{\mathbf{2}}\right)^{2}\right\}\left|\phi_{1}(\mathbf{q})\right|^{2}\right. \\
& \left.+\frac{1}{2 K}\left\{\left(\mathbf{q} \cdot \boldsymbol{\Delta}_{\mathbf{2}}\right)^{2}+\rho K\left(\mathbf{q} \cdot \boldsymbol{\Delta}_{\mathbf{1}}\right)^{2}\right\}\left|\phi_{2}(\mathbf{q})\right|^{2}\right] .
\end{aligned}
$$

One may notice that the fixed point Hamiltonian in Eq. 45 is invariant under $q_{x} \leftrightarrow q_{y}$ which implies that the fixed points under investigation do retain the full underlying symmetry of the lattice. This is different than the case we studied in Sec. III B. As before, the scaling relations show that any small value of $\rho$ is an attractive fixed point, we identify the $\rho=0$ fixed point as the unbound dislocation state, and $\rho>0$ as logarithmically bound dislocation states. Because of the full lattice symmetry of $\mathcal{H}_{*}$ there are now two nodal lines in the Brillouin zone when $\rho=0$.

For our later calculations we will ignore the cross terms in $\mathcal{H}_{0}\left(\phi_{1} \phi_{2}\right)$ as those terms contribute only at higher orders of $\xi^{2}$ (starting from $\xi^{4}$ ), and the calculation is greatly simplified because $\left\langle\phi_{1} \phi_{2}\right\rangle_{0}=0$ for the Hamiltonian in Eq. 44 when this is done. With this simplification, the propagators for the fields $\phi_{1}$ and $\phi_{2}$ calculated from Eq. 44 are

$$
\begin{aligned}
= & \frac{\left\langle\phi_{1}(-\mathbf{q}) \phi_{1}(\mathbf{q})\right\rangle_{0}}{\left(\mathbf{q} \cdot \boldsymbol{\Delta}_{\mathbf{1}}\right)^{2}+\rho K\left(\mathbf{q} \cdot \boldsymbol{\Delta}_{\mathbf{2}}\right)^{2}+\xi^{2}\left(\mathbf{q} \cdot \boldsymbol{\Delta}_{\mathbf{1}}\right)^{2}\left(\mathbf{q} \cdot \boldsymbol{\Delta}_{\mathbf{2}}\right)^{2}}, \\
= & \frac{\left\langle\phi_{2}(-\mathbf{q}) \phi_{2}(\mathbf{q})\right\rangle_{0}}{\left(\mathbf{q} \cdot \boldsymbol{\Delta}_{\mathbf{2}}\right)^{2}+\rho K\left(\mathbf{q} \cdot \boldsymbol{\Delta}_{\mathbf{1}}\right)^{2}+\xi^{2}\left(\mathbf{q} \cdot \boldsymbol{\Delta}_{\mathbf{1}}\right)^{2}\left(\mathbf{q} \cdot \boldsymbol{\Delta}_{\mathbf{2}}\right)^{2}} .
\end{aligned}
$$

With these we define the momentum shell integrals $\mathcal{L}_{\square}^{i}(\rho, \xi)$ for the the square lattice

$$
\begin{aligned}
& \mathcal{L}_{\square}^{1}(\rho, \xi) d \ell=K \int^{>} \frac{d^{2} \mathbf{q}}{(2 \pi)^{2}}\left(\mathbf{q} \cdot \boldsymbol{\Delta}_{\mathbf{2}}\right)^{2}\left\langle\phi_{1}(-\mathbf{q}) \phi_{1}(\mathbf{q})\right\rangle, \\
& \mathcal{L}_{\square}^{2}(\rho, \xi) d \ell=K \int^{>} \frac{d^{2} \mathbf{q}}{(2 \pi)^{2}}\left(\mathbf{q} \cdot \boldsymbol{\Delta}_{\mathbf{1}}\right)^{2}\left\langle\phi_{2}(-\mathbf{q}) \phi_{2}(\mathbf{q})\right\rangle .
\end{aligned}
$$

Note $\mathcal{L}_{\square}^{1}=\mathcal{L}_{\square}^{2} \equiv \mathcal{L}_{\square}$ because of the square symmetry. The scaling relation in Eq. 39 remains the same, provided we substitute $\mathcal{L} \rightarrow \mathcal{L}_{\square}^{1}+\mathcal{L}_{\square}^{2}=2 \mathcal{L}_{\square}$. The shell integral $\mathcal{L}_{\square}$ may be computed to yield

$$
\begin{aligned}
\mathcal{L}_{\square}(\rho, \xi) & =\frac{4 K \Lambda \pi}{\rho K+\xi^{2} \Lambda^{2}} \\
& +\frac{4 K \Lambda^{2}}{\sqrt{\rho K\left(1+\xi^{2} \Lambda^{2}\right)}} \arctan \left(\frac{\pi \sqrt{1+\xi^{2} \Lambda^{2}}}{\Lambda \sqrt{\rho K}}\right) \\
& -\frac{4 K \Lambda^{2}}{\left(\rho K+\xi^{2} \Lambda^{2}\right)^{3 / 2}} \arctan \left(\frac{\pi \sqrt{\rho K+\xi^{2} \Lambda^{2}}}{\Lambda}\right),
\end{aligned}
$$

where we have returned to unitless distances, $\Delta_{1,2}=$ $a_{0} \equiv 1$.

For small values of $\rho K$ we perform a Taylor series to obtain

$$
\mathcal{L}_{\square}(\rho, \xi) \approx \frac{K \Lambda^{2}}{2 \pi \sqrt{\rho K\left(1+\xi^{2} \Lambda^{2}\right)}} .
$$

We thus see that the scaling relation for $\rho$ in this formulation is identical to Eq. 39. The only caveat is that in our previous formulation, this relation held to all orders in $\xi^{2}$, whereas here there are corrections of $\mathcal{O}\left(\xi^{4}\right)$ and higher. In Sec. V we will present further results that follow from numerically integrating the scaling relations. 


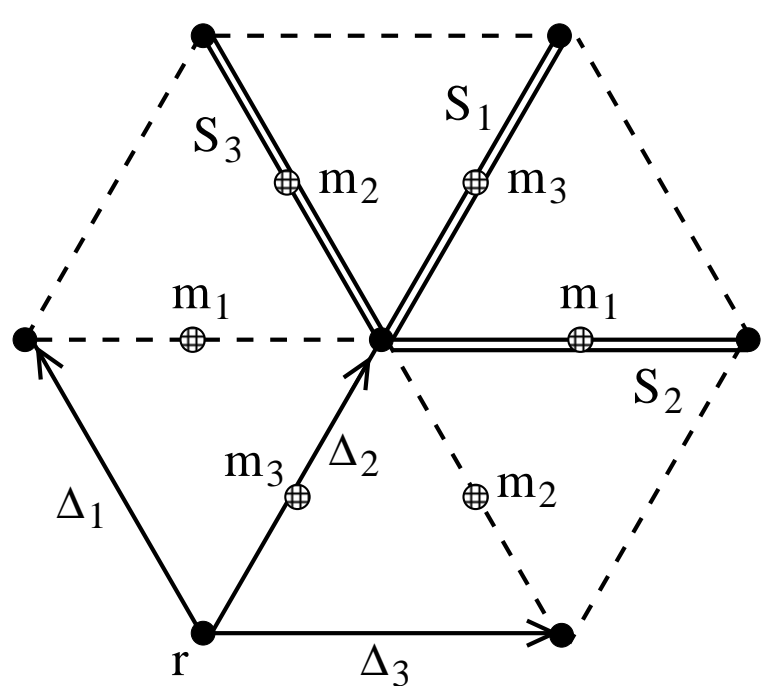

FIG. 8. Real lattice sites (r) (black filled circles) and bond center sites (patterned circles) used to represent $S_{1}, S_{2}$ and $S_{3}$ as differences for a triangular lattice. $\boldsymbol{\Delta}_{\mathbf{1}}, \boldsymbol{\Delta}_{\mathbf{2}}$ and $\boldsymbol{\Delta}_{\mathbf{3}}$ are vectors denoting nearest neighbor bonds. Note that the lines connecting the $m$ 's cross the bonds they define, but they are not perpendicular to them.

\section{B. Triangular Lattice}

We now consider our theoretical model on a triangular lattice, following the same procedure as in Sec. IV A. For our triangular lattice we have three bond variables $S_{1}, S_{2}$, and $S_{3}$ which are defined via three integer fields $m_{1}, m_{2}$ and $m_{3}$ (respecting the symmetry of the lattice)

$$
\begin{aligned}
& S_{1}(\mathbf{r})=m_{1}\left(\mathbf{r}+\boldsymbol{\Delta}_{\mathbf{3}} / 2+\boldsymbol{\Delta}_{\mathbf{1}}\right)-m_{1}\left(\mathbf{r}+\boldsymbol{\Delta}_{\mathbf{3}} / 2\right), \\
& S_{2}(\mathbf{r})=m_{2}\left(\mathbf{r}-\boldsymbol{\Delta}_{\mathbf{1}} / 2+\boldsymbol{\Delta}_{\mathbf{2}}\right)-m_{2}\left(\mathbf{r}-\boldsymbol{\Delta}_{\mathbf{1}} / 2\right), \\
& S_{3}(\mathbf{r})=m_{3}\left(\mathbf{r}+\boldsymbol{\Delta}_{\mathbf{2}} / 2\right)-m_{3}\left(\mathbf{r}+\boldsymbol{\Delta}_{\mathbf{2}} / 2-\boldsymbol{\Delta}_{\mathbf{3}}\right) .
\end{aligned}
$$

One may note that in Eqs. 50 the integer fields $m_{1,2,3}$ are not defined on the dual lattice sites as was done for the square lattice: in this case they are on the centers of the bonds, as illustrated in Fig. 8. The lines connecting the $m$ 's cross the bonds they define, but they are not perpendicular to them. The direction of the $\boldsymbol{\Delta}_{\mathbf{i}}$ 's are also shown in Fig. 8. Substituting Eqs. 50 in Eq. 5 we obtain the unperturbed Hamiltonian in momentum space

$$
\begin{aligned}
& \mathcal{H}_{\mathrm{VM}} \\
= & \frac{1}{N} \sum_{\mathbf{q}}\left\{\frac{1}{2 K}\left[\left|Q_{1} m_{1}(\mathbf{q})\right|^{2}+\left|Q_{2} m_{2}(\mathbf{q})\right|^{2}+\left|Q_{3} m_{3}(\mathbf{q})\right|^{2}\right]\right. \\
+ & \left.\frac{1}{2 h}\left|Q_{1} Q_{2} m_{1}(\mathbf{q})+Q_{2} Q_{3} m_{2}(\mathbf{q})+Q_{3} Q_{1} m_{3}(\mathbf{q})\right|^{2}\right\}, \quad(51)
\end{aligned}
$$

where $Q_{j}=1-e^{-i \mathbf{q} \cdot \boldsymbol{\Delta}_{\mathbf{j}}}$. As before we replace the integer fields $m_{1,2,3}$ with continuous fields $\phi_{1,2,3}$ and add in cosine terms. The cosine term that is analogous to $y_{a} \cos (2 \pi a)$ is

$$
\begin{aligned}
\mathcal{H}^{\prime} & =-y_{a} \sum_{\mathbf{r}}\left[\left(1-\cos \left\{2 \pi\left[\phi_{1}\left(\mathbf{r}+\boldsymbol{\Delta}_{\mathbf{2}}\right)-\phi_{1}(\mathbf{r})\right]\right\}\right)\right. \\
& +\left(1-\cos \left\{2 \pi\left[\phi_{2}\left(\mathbf{r}+\boldsymbol{\Delta}_{\mathbf{3}}\right)-\phi_{2}(\mathbf{r})\right]\right\}\right) \\
& \left.+\left(1-\cos \left\{2 \pi\left[\phi_{3}\left(\mathbf{r}+\boldsymbol{\Delta}_{\mathbf{1}}\right)-\phi_{3}(\mathbf{r})\right]\right\}\right)\right] .
\end{aligned}
$$

We now do a Fourier transform of the fields $m_{1,2,3}$ and expand the result in long wavelength limit $\phi_{i}\left(\mathbf{r}+\boldsymbol{\Delta}_{\mathbf{j}}\right)-$ $\phi_{i}(\mathbf{r}) \approx \frac{1}{N} \sum_{\mathbf{q}}\left(i \mathbf{q} \cdot \boldsymbol{\Delta}_{\mathbf{j}}\right) e^{i \mathbf{q} \cdot \mathbf{r}} \phi_{i}(\mathbf{q})$ where $i \neq j=1,2,3$. The resulting effective Hamiltonian is

$$
\begin{aligned}
\mathcal{H}_{\mathrm{eff}} & =\frac{1}{N} \sum_{\mathbf{q}}\left\{\frac { 1 } { 2 K } \left[\left(\mathbf{q} \cdot \boldsymbol{\Delta}_{\mathbf{1}}\right)^{2}\left|\phi_{1}(\mathbf{q})\right|^{2}\right.\right. \\
& \left.+\left(\mathbf{q} \cdot \boldsymbol{\Delta}_{\mathbf{2}}\right)^{2}\left|\phi_{2}(\mathbf{q})\right|^{2}+\left(\mathbf{q} \cdot \boldsymbol{\Delta}_{\mathbf{3}}\right)^{2}\left|\phi_{3}(\mathbf{q})\right|^{2}\right] \\
& +\frac{1}{2 h} \mid\left(\mathbf{q} \cdot \boldsymbol{\Delta}_{\mathbf{1}}\right)\left(\mathbf{q} \cdot \boldsymbol{\Delta}_{\mathbf{2}}\right) \phi_{1}(\mathbf{q}) \\
& \left.+\left(\mathbf{q} \cdot \boldsymbol{\Delta}_{\mathbf{2}}\right)\left(\mathbf{q} \cdot \boldsymbol{\Delta}_{\mathbf{3}}\right) \phi_{2}(\mathbf{q})+\left.\left(\mathbf{q} \cdot \boldsymbol{\Delta}_{\mathbf{3}}\right)\left(\mathbf{q} \cdot \boldsymbol{\Delta}_{\mathbf{1}}\right) \phi_{3}(\mathbf{q})\right|^{2}\right\} \\
-y_{a} \sum_{\mathbf{r}} & \left\{\left(1-\cos \left[\frac{2 \pi}{N} \sum_{\mathbf{q}}\left(i \mathbf{q} \cdot \boldsymbol{\Delta}_{\mathbf{2}}\right) e^{i \mathbf{q} \cdot \mathbf{r}} \phi_{1}(\mathbf{q})\right]\right)\right. \\
& +\left(1-\cos \left[\frac{2 \pi}{N} \sum_{\mathbf{q}}\left(i \mathbf{q} \cdot \boldsymbol{\Delta}_{\mathbf{3}}\right) e^{i \mathbf{q} \cdot \mathbf{r}} \phi_{2}(\mathbf{q})\right]\right) \\
& \left.+\left(1-\cos \left[\frac{2 \pi}{N} \sum_{\mathbf{q}}\left(i \mathbf{q} \cdot \boldsymbol{\Delta}_{\mathbf{1}}\right) e^{i \mathbf{q} \cdot \mathbf{r}} \phi_{3}(\mathbf{q})\right]\right)\right\} \\
& -y \sum_{\mathbf{r}}\left\{\left(1-\cos \left[2 \pi \phi_{1}(\mathbf{r})\right]\right)\right. \\
& \left.+\left(1-\cos \left[2 \pi \phi_{2}(\mathbf{r})\right]\right)+\left(1-\cos \left[2 \pi \phi_{3}(\mathbf{r})\right]\right)\right\} . \quad(53)
\end{aligned}
$$

In deriving Eq. 53 we took the long wavelength limit $Q_{j}=i \mathbf{q} \cdot \boldsymbol{\Delta}_{\mathbf{j}}(j=1,2,3)$. We may note that the above effective Hamiltonian is symmetric under interchanges of the $(1,2,3)$ indices, thus preserving the triangular lattice symmetry.

Our next step is to do the momentum shell RG on the effective Hamiltonian. As the procedure is identical to that discussed in Section IV A we present the main results. Expanding the cosine terms and absorbing the quadratic term into the unperturbed Hamiltonian, one finds

$$
\begin{aligned}
\mathcal{H}_{0}= & = \\
\frac{1}{N} \sum_{\mathbf{q}} & \left\{\frac { 1 } { 2 K } \left[\left\{\left(\mathbf{q} \cdot \boldsymbol{\Delta}_{\mathbf{1}}\right)^{2}+(\rho K)\left(\mathbf{q} \cdot \boldsymbol{\Delta}_{\mathbf{2}}\right)^{2}\right\}\left|\phi_{1}(\mathbf{q})\right|^{2}\right.\right. \\
& +\left\{\left(\mathbf{q} \cdot \boldsymbol{\Delta}_{\mathbf{2}}\right)^{2}+(\rho K)\left(\mathbf{q} \cdot \boldsymbol{\Delta}_{\mathbf{3}}\right)^{2}\right\}\left|\phi_{2}(\mathbf{q})\right|^{2} \\
& \left.+\left\{\left(\mathbf{q} \cdot \boldsymbol{\Delta}_{\mathbf{3}}\right)^{2}+(\rho K)\left(\mathbf{q} \cdot \boldsymbol{\Delta}_{\mathbf{1}}\right)^{2}\right\}\left|\phi_{3}(\mathbf{q})\right|^{2}\right]
\end{aligned}
$$




$$
\begin{aligned}
+\frac{\xi^{2}}{2 K} \mid & \left(\mathbf{q} \cdot \boldsymbol{\Delta}_{\mathbf{1}}\right)\left(\mathbf{q} \cdot \boldsymbol{\Delta}_{\mathbf{2}}\right) \phi_{1}(\mathbf{q}) \\
+ & \left(\mathbf{q} \cdot \boldsymbol{\Delta}_{\mathbf{2}}\right)\left(\mathbf{q} \cdot \boldsymbol{\Delta}_{\mathbf{3}}\right) \phi_{2}(\mathbf{q}) \\
+ & \left.\left.\left(\mathbf{q} \cdot \boldsymbol{\Delta}_{\mathbf{3}}\right)\left(\mathbf{q} \cdot \boldsymbol{\Delta}_{\mathbf{1}}\right) \phi_{3}(\mathbf{q})\right|^{2}\right\} .
\end{aligned}
$$

Rearranging the terms in Eq. 54 we get,

$$
\begin{aligned}
\mathcal{H}_{0} & =\frac{1}{N} \sum_{\mathbf{q}}\left\{\frac { 1 } { 2 K } \left[\left\{\left(\mathbf{q} \cdot \boldsymbol{\Delta}_{\mathbf{1}}\right)^{2}+\rho K\left(\mathbf{q} \cdot \boldsymbol{\Delta}_{\mathbf{2}}\right)^{2}\right.\right.\right. \\
& \left.+\xi^{2}\left(\mathbf{q} \cdot \boldsymbol{\Delta}_{\mathbf{1}}\right)^{2}\left(\mathbf{q} \cdot \boldsymbol{\Delta}_{\mathbf{2}}\right)^{2}\right\}\left|\phi_{1}(\mathbf{q})\right|^{2} \\
& +\left\{\left(\mathbf{q} \cdot \boldsymbol{\Delta}_{\mathbf{2}}\right)^{2}+\rho K\left(\mathbf{q} \cdot \boldsymbol{\Delta}_{\mathbf{3}}\right)^{2}\right. \\
& \left.+\xi^{2}\left(\mathbf{q} \cdot \boldsymbol{\Delta}_{\mathbf{2}}\right)^{2}\left(\mathbf{q} \cdot \boldsymbol{\Delta}_{\mathbf{3}}\right)^{2}\right\}\left|\phi_{2}(\mathbf{q})\right|^{2} \\
& +\left\{\left(\mathbf{q} \cdot \boldsymbol{\Delta}_{\mathbf{3}}\right)^{2}+\rho K\left(\mathbf{q} \cdot \boldsymbol{\Delta}_{\mathbf{1}}\right)^{2}\right. \\
& \left.\left.+\xi^{2}\left(\mathbf{q} \cdot \boldsymbol{\Delta}_{\mathbf{3}}\right)^{2}\left(\mathbf{q} \cdot \boldsymbol{\Delta}_{\mathbf{1}}\right)^{2}\right\}\left|\phi_{3}(\mathbf{q})\right|^{2}\right] \\
& +\frac{\xi^{2}}{K}\left[\left(\mathbf{q} \cdot \boldsymbol{\Delta}_{\mathbf{1}}\right)\left(\mathbf{q} \cdot \boldsymbol{\Delta}_{\mathbf{2}}\right)^{2}\left(\mathbf{q} \cdot \boldsymbol{\Delta}_{\mathbf{3}}\right) \phi_{1}(\mathbf{q}) \phi_{2}(\mathbf{q})\right. \\
& +\left(\mathbf{q} \cdot \boldsymbol{\Delta}_{\mathbf{1}}\right)\left(\mathbf{q} \cdot \boldsymbol{\Delta}_{\mathbf{2}}\right)\left(\mathbf{q} \cdot \boldsymbol{\Delta}_{\mathbf{3}}\right)^{2} \phi_{2}(\mathbf{q}) \phi_{3}(\mathbf{q}) \\
& \left.\left.+\left(\mathbf{q} \cdot \boldsymbol{\Delta}_{\mathbf{1}}\right)^{2}\left(\mathbf{q} \cdot \boldsymbol{\Delta}_{\mathbf{2}}\right)\left(\mathbf{q} \cdot \boldsymbol{\Delta}_{\mathbf{3}}\right) \phi_{1}(\mathbf{q}) \phi_{3}(\mathbf{q})\right]\right\} .
\end{aligned}
$$

Once again, the generic fixed point Hamiltonian is $y_{2 n \geq 4}=0, \xi^{2}=0$, and

$$
\begin{aligned}
\mathcal{H}_{*} & =\frac{1}{N} \sum_{\mathbf{q}} \frac{1}{2 K}\left[\left\{\left(\mathbf{q} \cdot \boldsymbol{\Delta}_{\mathbf{1}}\right)^{2}+\rho K\left(\mathbf{q} \cdot \boldsymbol{\Delta}_{\mathbf{2}}\right)^{2}\right\}\left|\phi_{1}(\mathbf{q})\right|^{2}\right. \\
& +\left\{\left(\mathbf{q} \cdot \boldsymbol{\Delta}_{\mathbf{2}}\right)^{2}+\rho K\left(\mathbf{q} \cdot \boldsymbol{\Delta}_{\mathbf{3}}\right)^{2}\right\}\left|\phi_{2}(\mathbf{q})\right|^{2} \\
& \left.+\left\{\left(\mathbf{q} \cdot \boldsymbol{\Delta}_{\mathbf{3}}\right)^{2}+\rho K\left(\mathbf{q} \cdot \boldsymbol{\Delta}_{\mathbf{1}}\right)^{2}\right\}\left|\phi_{3}(\mathbf{q})\right|^{2}\right]
\end{aligned}
$$

Because the fixed point Hamiltonian in Eq. 56 captures the triangular symmetry of the lattice, there are three nodal lines in the Brillouin zone for $\rho=0$. As before we identify this as the unbound dislocation phase. $\rho>0$ again corresponds to a bound dislocation phase.

For simplicity of our further calculations we will ignore the cross terms $\left(\phi_{1} \phi_{2}, \phi_{2} \phi_{3}, \phi_{1} \phi_{3}\right)$ in $\mathcal{H}_{0}$ as those terms contribute only at higher orders of $\xi^{2}$. In that case the propagators of Eq. 55 can be easily evaluated, yielding

$$
\begin{aligned}
= & \frac{\left\langle\phi_{1}(-\mathbf{q}) \phi_{1}(\mathbf{q})\right\rangle_{0}}{\left(\mathbf{q} \cdot \boldsymbol{\Delta}_{\mathbf{1}}\right)^{2}+\rho K\left(\mathbf{q} \cdot \boldsymbol{\Delta}_{\mathbf{2}}\right)^{2}+\xi^{2}\left(\mathbf{q} \cdot \boldsymbol{\Delta}_{\mathbf{1}}\right)^{2}\left(\mathbf{q} \cdot \boldsymbol{\Delta}_{\mathbf{2}}\right)^{2}}, \\
= & \frac{\left\langle\phi_{2}(-\mathbf{q}) \phi_{2}(\mathbf{q})\right\rangle_{0}}{\left(\mathbf{q} \cdot \boldsymbol{\Delta}_{\mathbf{2}}\right)^{2}+\rho K\left(\mathbf{q} \cdot \boldsymbol{\Delta}_{\mathbf{3}}\right)^{2}+\xi^{2}\left(\mathbf{q} \cdot \boldsymbol{\Delta}_{\mathbf{2}}\right)^{2}\left(\mathbf{q} \cdot \boldsymbol{\Delta}_{\mathbf{3}}\right)^{2}},
\end{aligned}
$$

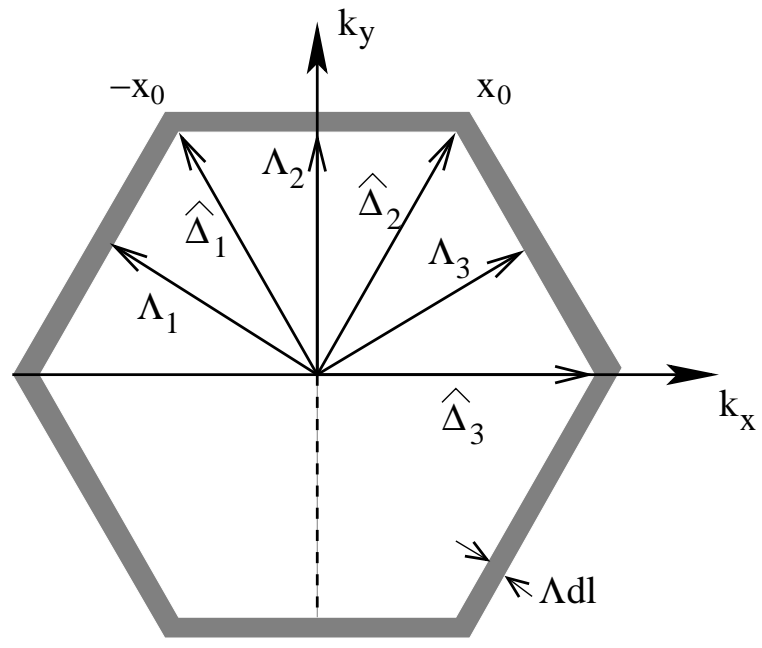

FIG. 9. Momentum shell of width $\Lambda d \ell$ in reciprocal space for the triangular lattice. $\boldsymbol{\Lambda}_{1}, \boldsymbol{\Lambda}_{2}$, and $\boldsymbol{\Lambda}_{3}$ are equal length vectors of length $2 \pi /(\sqrt{3}) . \hat{\Delta}_{1}, \hat{\Delta}_{2}$ and $\hat{\Delta}_{3}$ represent directions of bond vectors of length $a_{0}(\equiv 1)$ in Fig. 8 .

$$
=\frac{\left\langle\phi_{3}(-\mathbf{q}) \phi_{3}(\mathbf{q})\right\rangle_{0}}{\left(\mathbf{q} \cdot \boldsymbol{\Delta}_{\mathbf{3}}\right)^{2}+\rho K\left(\mathbf{q} \cdot \boldsymbol{\Delta}_{\mathbf{1}}\right)^{2}+\xi^{2}\left(\mathbf{q} \cdot \boldsymbol{\Delta}_{\mathbf{3}}\right)^{2}\left(\mathbf{q} \cdot \boldsymbol{\Delta}_{\mathbf{1}}\right)^{2}} .
$$

The momentum shell integrals that we need to evaluate for the scaling equation are

$$
\begin{aligned}
& \mathcal{L}_{\triangle}^{1} d \ell=K \int^{>} \frac{d^{2} \mathbf{q}}{(2 \pi)^{2}}\left(\mathbf{q} \cdot \boldsymbol{\Delta}_{\mathbf{2}}\right)^{2}\left\langle\phi_{1}(-\mathbf{q}) \phi_{1}(\mathbf{q})\right\rangle, \\
& \mathcal{L}_{\triangle}^{2} d \ell=K \int^{>} \frac{d^{2} \mathbf{q}}{(2 \pi)^{2}}\left(\mathbf{q} \cdot \boldsymbol{\Delta}_{\mathbf{3}}\right)^{2}\left\langle\phi_{2}(-\mathbf{q}) \phi_{2}(\mathbf{q})\right\rangle, \\
& \mathcal{L}_{\triangle}^{3} d \ell=K \int^{>} \frac{d^{2} \mathbf{q}}{(2 \pi)^{2}}\left(\mathbf{q} \cdot \boldsymbol{\Delta}_{\mathbf{1}}\right)^{2}\left\langle\phi_{3}(-\mathbf{q}) \phi_{3}(\mathbf{q})\right\rangle .
\end{aligned}
$$

Because of the lattice symmetry $\mathcal{L}_{\triangle}^{1}=\mathcal{L}_{\triangle}^{2}=\mathcal{L}_{\triangle}^{3} \equiv \mathcal{L}_{\triangle}$. The scaling relation in Eq. 39 again remains the same provided we replace $\mathcal{L}$ with $3 \mathcal{L}_{\triangle}$. As written, the integral for $\mathcal{L}_{\triangle}$ has to be done numerically over the momentum shell [shaded portion in Fig. 9].

Alternatively, an approximate analytical result of the integral can be derived by first doing a Taylor series expansion with respect to $\xi^{2}$ in the denominators of Eqs. 57 and then performing the integration. The result is shown in Appendix B. For small values of $\rho$, Eqs. 63, 66, 67 can be expanded to obtain an approximate result for $\mathcal{L}_{\triangle}$ which is

$$
\mathcal{L}_{\triangle}(\rho, \xi) \approx \frac{\sqrt{3} \Lambda^{2} K}{4 \pi \sqrt{\rho K}}\left[1-\frac{3}{8} \xi^{2} \Lambda^{2}\right] .
$$

This result shows that, as before, the shell integral diverges as $\rho \rightarrow 0$. Once again, the scaling relation for $\rho$ is identical to Eq. 39, with $\mathcal{L} \rightarrow 3 \mathcal{L}_{\triangle}$.

Finally, the evaluation of the constants $\Lambda$ and $x_{0}$ which define the shell geometry in Fig. 9 are slightly involved, so we explicitly show how to evaluate them. We 
take the real space basis vectors of the triangular lattice to be $\mathbf{R}_{\mathbf{1}}=a_{0} \hat{\mathbf{x}}$ (along $\hat{\Delta}_{3}$ in Fig. 8) and $\mathbf{R}_{\mathbf{2}}=$ $-\left(a_{0} / 2\right) \hat{\mathbf{x}}+\left(\sqrt{3} a_{0} / 2\right) \hat{\mathbf{y}}$ (along $\hat{\Delta}_{1}$ in Fig. 8 ). Then the basis vectors of the reciprocal lattice satifying the prop$\operatorname{erty} \mathbf{G}_{\mathbf{i}} \cdot \mathbf{R}_{\mathbf{j}}=2 \pi \delta_{i j}$ are $\mathbf{G}_{\mathbf{1}}=\left(2 \pi / a_{0}\right) \hat{\mathbf{x}}+\left(2 \pi / \sqrt{3} a_{0}\right) \hat{\mathbf{y}}$ and $\mathbf{G}_{\mathbf{2}}=\left(4 \pi / \sqrt{3} a_{0}\right) \hat{\mathbf{y}} . \Lambda$ is the distance from the Brillouin zone center to the middle of one of the edges, and has length equal to half the length of the reciprocal basis vector $\mathbf{G}_{\mathbf{2}}$ (See Fig. 9). Thus $\Lambda=2 \pi /\left(\sqrt{3} a_{0}\right)$. From the momentum shell diagram we find that the half-width of the Brillouin zone edge is $x_{0}=\Lambda / \sqrt{3}=2 \pi /\left(3 a_{0}\right)$. $\Delta_{1}, \Delta_{2}, \Delta_{3}$ are all equal to the lattice spacing $a_{0}$ which we have chosen to be of unit length.

\section{DISCUSSION: CRITICAL BEHAVIOR AND PHASE DIAGRAM}

Because the scaling relation for $\rho$ is essentially identical (up to numerical factors) for the calculations incorporating the lattice symmetry and for the approximations used in Section III C, it is no surprise that the integration of Eq. 39 gives results that are nearly identical to what we found previously. Fig. 10 illustrates typical results for the square and triangular lattice case. As before, we find flows that either accumulate at the $\rho=0$ point, which we identify with the unbound dislocation phase, or flows that end at $\rho>0$, which represent logarithmically bound dislocation pairs. As discussed above, this is a remarkable fixed point structure: there are no flows whose trajectory change discontinuously as the phase boundary is crossed; i.e., there are no relevant directions (in the RG sense) leading away from the $\rho=0$ fixed point. One can consider operators other than the ones we have discussed explicitly - cosines of higher order derivatives of the $\phi$ 's, analogous to, for example, Eq. $41-$ but it is not hard to convince oneself that such operators will be more strongly irrelevant than those with which we have worked. Cosines of integrals of the $\phi$ 's are highly irrelevant as well.

In the absence of any such relevant directions, standard RG theory ${ }^{7}$ tells us that the free energy $[F(\ell \rightarrow \infty)]$ is not singular, and one should not expect to find signals of a phase transition in thermodynamic quantities. Despite this, there are some critical properties associated with the deconfinement transition as we have found it. For example, the screening length $\ell_{\mathrm{scr}}=a_{0} e^{\ell^{*}}$, defined by the scale $\ell^{*}$ at which a flow going to the unbound dislocation fixed point first strikes the $\rho=0$ axis (discussed in Section III C), diverges as the confinement transition is approached. Eq. 39 suggests this divergence is a power law in $\left(\xi_{0}^{2}-\xi_{c r}^{2}\right)$, which may be confirmed by examining the flows in detail. The behavior of $\ell_{\text {scr }}$ as a function of $\xi_{0}^{2}$ is illustrated in Fig. 11, where it is apparent that $\ell_{s c r}$ diverges with a critical exponent $-1 / 2$ as $\xi_{0}^{2} \rightarrow \xi_{c r}^{2}$ from below. More explicitly, for fixed $K$ this indicates $\ell_{s c r} \sim\left|h-h_{c}\right|^{-1 / 2}$. The same power law is obtained for the triangular lattice.
Another parameter of interest is the string tension $\rho$, which controls the effective logarithmic interaction between vortices. Fig. 12 illustrates how this behaves as $\left|\xi_{0}^{2}-\xi_{c r}^{2}\right| \rightarrow 0$. It is apparent that $\rho$ vanishes quadratically with this difference; or, equivalently, $\rho \sim\left|h-h_{c}\right|^{2}$. In principle the parameter $\rho$ can be measured if one can create a vortex-antivortex pair at controlled locations in the system, and measure the force required to separate them. From the discussion in Section III B (see Eq. 37), it is clear that for a separation $R$, the force required will have the form $(1+C) / R$, with $C$ diverging when $\rho=0$. The divergence indicates a change in behavior from a force that falls off with distance to one that remains finite for any $R$, reflecting the presence of a string connecting the two vortices. We note that the diverging coefficient $C$ does not truly indicate a diverging force; even when $\rho>0$, for small separations the force will be independent of separation, up to a characteristic crossover separation $R_{c} \sim 1 / \sqrt{\rho K}$, where the cutoff in the denominator of Eq. 37 becomes apparent and the $1 / R$ force sets in. Near the transition, the $(1+C) / R$ behavior only begins at very large $R$; the effective force between the vortices is thus never large.

Finally, we discuss the reasoning leading to the proposed phase diagram illustrated in Fig. 1. For the purposes of this discussion, we consider $K$ near its dual point ( $K \approx 1 / 2 \pi$ ), which it should be noted for $h=0$ is well into the unbound vortex phase. For large $h$, the vortices will instead be linearly bound, even at this small value of $K$. As we know by now, with increasing $1 / h$, there is a transition from an unbound to a logarithmically bound dislocation phase. This may alternatively be understood as a transition from linearly confined to logarithmically bound vortices, as described in the last subsection. Since $K$ is near the dual point $(K \approx 1 / 2 \pi)$, the duality in the model tells us there must be another transition at large $1 / h$ as $E_{c}$ increases from zero, representing a transition from unbound to logarithmically bound vortices. The dislocation deconfinement transition for which we developed the RG analysis is represented as the large $E_{c}$ limit of the left transition line, and its dual is the large $1 / h$ limit of the right transition line. For vortices, we know that unbinding becomes increasingly difficult with increasing $h$, so that a smaller $E_{c}$ is presumably required; this is why the phase boundaries move toward the axes as they approach the origin. We emphasize that the precise behavior of these phase boundaries as $1 / h$ and $E_{c}$ decrease is unclear, since our approach requires one (but not both) of these to be large in order to perform a controlled calculation. The diagram shown in Fig. 1 is the simplest that is consistent with what we have found in our calculations. As discussed above, the existence of the three phases is supported by numerical simulation studies $^{14}$. 


\section{CONCLUSION}

We have performed a renormalization group analysis to study vortex unbinding for the classical two dimensional $X Y$ model in a magnetic field on square and triangular lattices. Our analysis shows that if one starts at high temperature and large magnetic field, vortices in the model unbind as the field is lowered in a two-step process: first strings of overturned spins proliferate, and then vortices unbind. The proliferated string phase may be understood as one in which the vortices are logarithmically bound, whereas in the high field phase they are linearly confined. This transition has an alternate description in terms of domain walls and screw dislocations, at which the dislocations deconfine. The vortex deconfinement transition was shown to be dual to this. Both transitions are remarkably continuous, but they are not of the Kosterlitz-Thouless type. Finally, the unbound vortex fixed point was shown to contain a set of nodal lines which are lines of zero energy modes, reflecting the symmetry of the lattice.

\section{APPENDIX A}

In this Appendix we display the explicit form the shell integral in Eq. 34. Defining the parameters $A=1+B$, $B=K \rho+\Lambda \xi^{2}$, one finds

$$
\begin{aligned}
\mathcal{L}(\rho, \xi)=\frac{2 K \Lambda^{2}}{\pi^{2}} & \left\{\frac{1}{\sqrt{A^{2}-4 K \rho \Lambda^{2} \xi^{2}}}\right. \\
& \times\left[\frac{R_{+}-1}{R_{+}} \arctan \frac{1}{\sqrt{R_{+}}}\right. \\
& \left.-\frac{R_{-}-1}{R_{-}} \arctan \frac{1}{\sqrt{R_{-}}}\right] \\
& \left.+\frac{1}{B}\left[1-\frac{1}{\sqrt{A B}} \arctan \sqrt{\frac{B}{A}}\right]\right\} .
\end{aligned}
$$

In Eq. 60, the parameters $R_{ \pm}$are given by

$$
R_{ \pm}=\left\{A \pm \sqrt{A^{2}-4 K \rho \Lambda^{2} \xi^{2}}\right\} / 2 \Lambda^{2} \xi^{2} .
$$

Eq. 60 can be expanded for small $\rho$ to yield the result

$$
\begin{aligned}
\mathcal{L}(\rho, \xi) & =\frac{2 K \Lambda^{2}}{\pi^{2}}\left\{\frac{\pi}{2} \frac{1}{\sqrt{K \rho\left(1+\xi^{2} \Lambda^{2}\right)}}+\frac{1}{\xi^{2} \Lambda^{2}}\right. \\
& -\frac{1}{1+\xi^{2} \Lambda^{2}}+\left[\frac{1}{\Lambda \xi\left(1+\xi^{2} \Lambda^{2}\right)}\right. \\
& \left.\left.-\frac{1}{\Lambda^{3} \xi^{3} \sqrt{1+\xi^{2} \Lambda^{2}}}\right] \arctan \frac{\Lambda \xi}{\left(1+\Lambda^{2} \xi^{2}\right)^{3 / 2}}\right\} \\
& +\mathcal{O}(\sqrt{\rho})
\end{aligned}
$$

For small values of $\rho$, the first term in Eq. 61 dominates the RG flows.

\section{APPENDIX B}

In this Appendix we show the approximate analytical results for the following shell integral (See Eqs. 58) with $\mu=\rho K$

$$
\begin{aligned}
& \mathcal{L}_{\triangle}(\mu, \xi) d \ell=K \int_{\text {shell }} \frac{d^{2} \mathbf{q}}{(2 \pi)^{2}} \\
\times & \frac{\left(\mathbf{q} \cdot \boldsymbol{\Delta}_{\mathbf{2}}\right)^{2}}{\left(\mathbf{q} \cdot \boldsymbol{\Delta}_{\mathbf{1}}\right)^{2}+\mu\left(\mathbf{q} \cdot \boldsymbol{\Delta}_{\mathbf{2}}\right)^{2}+\xi^{2}\left(\mathbf{q} \cdot \boldsymbol{\Delta}_{\mathbf{1}}\right)^{2}\left(\mathbf{q} \cdot \boldsymbol{\Delta}_{\mathbf{2}}\right)^{2}} .
\end{aligned}
$$

To evaluate this integral we expand the denominator for small $\xi^{2}$. The integral now looks as,

$$
\mathcal{L}_{\triangle}(\mu, \xi) d \ell=\mathcal{L}_{\triangle}^{(a)}(\mu) d \ell+\xi^{2} \frac{d}{d \mu} \mathcal{L}_{\triangle}^{(b)}(\mu) d \ell,
$$

where,

$$
\begin{aligned}
& \mathcal{L}_{\triangle}^{(a)} d \ell=K \int_{\text {shell }} \frac{d^{2} \mathbf{q}}{(2 \pi)^{2}} \frac{\left(\mathbf{q} \cdot \boldsymbol{\Delta}_{\mathbf{2}}\right)^{2}}{\left(\mathbf{q} \cdot \boldsymbol{\Delta}_{\mathbf{1}}\right)^{2}+\mu\left(\mathbf{q} \cdot \boldsymbol{\Delta}_{\mathbf{2}}\right)^{2}} \\
& \mathcal{L}_{\triangle}^{(b)} d \ell=K \int_{\text {shell }} \frac{d^{2} \mathbf{q}}{(2 \pi)^{2}} \frac{\left(\mathbf{q} \cdot \boldsymbol{\Delta}_{\mathbf{1}}\right)^{2}\left(\mathbf{q} \cdot \boldsymbol{\Delta}_{\mathbf{2}}\right)^{2}}{\left(\mathbf{q} \cdot \boldsymbol{\Delta}_{\mathbf{1}}\right)^{2}+\mu\left(\mathbf{q} \cdot \boldsymbol{\Delta}_{\mathbf{2}}\right)^{2}}
\end{aligned}
$$

We now display the results of the above integrals.

$$
\begin{aligned}
& 2 \pi^{2} \mathcal{L}_{\triangle}^{(a)}=\frac{8 K \Lambda x_{0}}{1+4 \mu} \\
& -\frac{4 K \sqrt{3} \Lambda^{2}}{(1+4 \mu)^{2}} \ln \left(\frac{3 \Lambda^{2}+2 \sqrt{3} \Lambda x_{0}+x_{0}^{2}(1+4 \mu)}{3 \Lambda^{2}-2 \sqrt{3} \Lambda x_{0}+x_{0}^{2}(1+4 \mu)}\right) \\
& +\frac{2 K \sqrt{3} \Lambda^{2}(1-4 \mu)}{\sqrt{\mu}(1+4 \mu)^{2}} \arctan \left(\frac{4 \sqrt{3} \Lambda x_{0} \sqrt{\mu}}{3 \Lambda^{2}-x_{0}^{2}(1+4 \mu)}\right) \\
& +\frac{2 K \Lambda x_{0}}{1+\mu}+\frac{2 \sqrt{3} K \Lambda^{2}}{(1+\mu)^{2}} \\
& \times \ln \left(\frac{3 \Lambda^{2}(1+\mu)+2 \sqrt{3} \Lambda x_{0}(\mu-1)+x_{0}^{2}(1+\mu)}{3 \Lambda^{2}(1+\mu)-2 \sqrt{3} \Lambda x_{0}(\mu-1)+x_{0}^{2}(1+\mu)}\right) \\
& +\frac{2 K \sqrt{3} \Lambda^{2}(1-\mu)}{\sqrt{\mu}(1+\mu)^{2}} \arctan \left(\frac{4 \sqrt{3} \Lambda x_{0} \sqrt{\mu}}{(1+\mu)\left(3 \Lambda^{2}-x_{0}^{2}\right)}\right) \\
& +\frac{2 K \Lambda x_{0}}{4+\mu} \\
& +\frac{4 \sqrt{3} \Lambda^{2}}{(4+\mu)^{2}} \ln \left(\frac{3 \Lambda^{2} \mu+2 \sqrt{3} \Lambda x_{0} \mu+x_{0}^{2}(4+\mu)}{3 \Lambda^{2} \mu-2 \sqrt{3} \Lambda x_{0} \mu+x_{0}^{2}(4+\mu)}\right) \\
& -\frac{2 K \sqrt{3} \Lambda^{2}(\mu-4)}{\sqrt{\mu}(4+\mu)^{2}} \arctan \left(\frac{4 \sqrt{3} \Lambda x_{0} \sqrt{\mu}}{3 \Lambda^{2} \mu-x_{0}^{2}(4+\mu)}\right) \text {. } \\
& 2 \pi^{2} \frac{d \mathcal{L}_{\triangle}^{(b)}}{d \mu} \\
& =-\frac{8 K \Lambda \Delta^{2} x_{0}^{3}}{3(4 \mu+1)^{2}}-\frac{24 K \Lambda^{3} \Delta^{2}\left(16 \mu^{2}-32 \mu+3\right) x_{0}}{(4 \mu+1)^{4}} \\
& +\frac{24 \sqrt{3} K \Lambda^{4} \Delta^{2}\left(32 \mu^{2}-20 \mu+1\right)}{(4 \mu+1)^{5}}
\end{aligned}
$$




$$
\begin{aligned}
& \times \ln \left(\frac{3 \Lambda^{2}+2 \sqrt{3} \Lambda x_{0}+x_{0}^{2}(4 \mu+1)}{3 \Lambda^{2}-2 \sqrt{3} \Lambda x_{0}+x_{0}^{2}(4 \mu+1)}\right) \\
& +\frac{1152 K \Lambda^{5} \Delta^{2} \mu(4 \mu-1) x_{0}^{3}}{(4 \mu+1)^{4}\left[\left[3 \Lambda^{2}+x_{0}^{2}(4 \mu+1)\right]^{2}-12 \Lambda^{2} x_{0}^{2}\right]} \\
& +\left(\frac{3 \sqrt{3} K \Lambda^{4} \Delta^{2}\left(192 \mu^{3}-560 \mu^{2}+100 \mu-1\right)}{\sqrt{\mu}(4 \mu+1)^{5}}\right) \\
& \times \arctan \left(\frac{4 \sqrt{3} \Lambda \sqrt{\mu} x_{0}}{3 \Lambda^{2}-x_{0}^{2}(4 \mu+1)}\right) \\
& -\frac{36 K \Lambda^{5} \Delta^{2}\left[3 \Lambda^{2}+x_{0}^{2}(4 \mu-1)\right]\left[16 \mu^{2}-24 \mu+1\right] x_{0}}{(4 \mu+1)^{4}\left[9 \Lambda^{4}+6 \Lambda^{2} x_{0}^{2}(4 \mu-1)+x_{0}^{4}(4 \mu+1)^{2}\right]} \\
& -\frac{K \Lambda \Delta^{2} x_{0}^{3}}{6(\mu+1)^{2}}-\frac{3 K \Lambda^{3} \Delta^{2}\left(\mu^{2}-30 \mu+17\right) x_{0}}{(\mu+1)^{4}} \\
& -\frac{12 \sqrt{3} K \Lambda^{4} \Delta^{2}\left(2 \mu^{2}-5 \mu+1\right)}{(\mu+1)^{5}} \\
& \times \ln \left(\frac{3 \Lambda^{2}(1+\mu)+2 \sqrt{3} \Lambda(\mu-1) x_{0}+x_{0}^{2}(\mu+1)}{3 \Lambda^{2}(1+\mu)-2 \sqrt{3} \Lambda(\mu-1) x_{0}+x_{0}^{2}(\mu+1)}\right) \\
& +\frac{288 K \Lambda^{5} \Delta^{2}\left(3 \Lambda^{2}+x_{0}^{2}\right) x_{0} \mu(\mu-1)}{(\mu+1)^{4}\left[\left[3 \Lambda^{2}(1+\mu)+(\mu+1) x_{0}^{2}\right]^{2}-12 \Lambda^{2} x_{0}^{2}(\mu-1)^{2}\right]} \\
& +\left(\frac{12 \sqrt{3} K \Lambda^{4} \Delta^{2}\left(3 \mu^{3}-35 \mu^{2}+25 \mu-1\right)}{\sqrt{\mu}(\mu+1)^{5}}\right) \\
& \times \arctan \left(\frac{4 \sqrt{3} \Lambda \sqrt{\mu} x_{0}}{(1+\mu)\left(3 \Lambda^{2}-x_{0}^{2}\right)}\right) \\
& +\left\{144 K \Lambda^{5} \Delta^{2}\left(3 \Lambda^{2}-x_{0}^{2}\right) x_{0}(\mu-1)\left(\mu^{2}-6 \mu+1\right)\right\} \\
& \times\left\{( \mu + 1 ) ^ { 4 } \left[9 \Lambda^{4}-6 \Lambda^{2} x_{0}^{2}+x_{0}^{4}(\mu+1)^{2}+18 \Lambda^{4} \mu\right.\right. \\
& \left.\left.+36 \Lambda^{2} x_{0}^{2} \mu-6 \Lambda^{2} x_{0}^{2} \mu^{2}+9 \Lambda^{4} \mu^{2}\right]\right\}^{-1} \\
& -\frac{2 K \Lambda \Delta^{2} x_{0}^{3}}{3(\mu+4)^{2}}+\frac{144 K \Lambda^{3} \Delta^{2}(\mu-4) x_{0}}{(\mu+4)^{4}} \\
& -\frac{48 \sqrt{3} K \Lambda^{4} \Delta^{2}\left(\mu^{2}-10 \mu+8\right)}{(\mu+4)^{5}} \\
& \times \ln \left(\frac{3 \Lambda^{2} \mu+2 \sqrt{3} \Lambda \mu x_{0}+x_{0}^{2}(\mu+4)}{3 \Lambda^{2} \mu-2 \sqrt{3} \Lambda \mu x_{0}+x_{0}^{2}(\mu+4)}\right) \\
& +\frac{1152 K \Lambda^{5} \Delta^{2} \mu(\mu-4) x_{0}^{3}}{(\mu+4)^{4}\left[\left[3 \Lambda^{2} \mu+(\mu+4) x_{0}^{2}\right]^{2}-12 \Lambda^{2} \mu^{2} x_{0}^{2}\right]} \\
& +\left(\frac{3 \sqrt{3} K \Lambda^{4} \Delta^{2}\left(3 \mu^{3}-140 \mu^{2}+400 \mu-64\right)}{\sqrt{\mu}(\mu+4)^{5}}\right) \\
& \times \arctan \left(\frac{4 \sqrt{3} \Lambda \sqrt{\mu} x_{0}}{3 \Lambda^{2} \mu-x_{0}^{2}(\mu+4)}\right) \\
& +\left\{36 K \Lambda^{5} \Delta^{2}\left[3 \Lambda^{2} \mu+x_{0}^{2}(4-\mu)\right] x_{0}\left[\mu^{2}-24 \mu+16\right]\right\} \\
& \times\left\{( \mu + 4 ) ^ { 4 } \left[9 \Lambda^{4} \mu^{2}+24 \Lambda^{2} \mu x_{0}^{2}-6 \Lambda^{2} \mu^{2} x_{0}^{2}\right.\right. \\
& \left.\left.+x_{0}^{4}(\mu+4)^{2}\right]\right\}^{-1} \text {. }
\end{aligned}
$$

\section{ACKNOWLEDGMENTS}

The authors would like to thank many colleagues for helpful discussions and suggestions, particularly Joseph P. Straley, Ganpathy Murthy, Donald Priour, Andy Lau, and Ramin Golestanian. This work was supported by NSF Grant No. DMR-0108451.

${ }^{1}$ D. R. Nelson, Defects and Geometry in Condensed Matter Physics (Cambridge University Press, New York, 2002).

${ }^{2}$ J. M. Kosterlitz and D. Thouless, J. Phys. C: Solid State Phys. 6, 1181 (1973); J. M. Kosterlitz, J. Phys. C: Solid State Phys. 7, 1046 (1974).

${ }^{3}$ P. M. Chaikin and T. C. Lubensky, Principles of Condensed Matter Physics (Cambridge University Press, New York, 1995).

${ }^{4}$ A. O. Gogolin, A. A. Nersesyan, and A. M. Tsvelik, Bosonization and Stronly Correlated Systems (Cambridge University Press, Cambridge, 1998).

${ }^{5}$ S. A. Kivelson, E. Fradkin, and V. J. Emery, Nature (London) 393, 550, 1998; V. J. Emery, E. Fradkin, S. A. Kivelson, and T. C. Lubensky, Phys. Rev. Lett. 85, 2160 (2000).

${ }^{6}$ H. A. Fertig, Phys. Rev. Lett. 82, 3693 (1999); Hangmo Yi, H. A. Fertig, and R. Côté, Phys. Rev. Lett. 85, 4156 (2000).

7 J. Cardy, Scaling and Renormalization in Statistical Physics (Cambridge University Press, New York, 1996).

${ }^{8}$ J. V. José, L. P. Kadanoff, S. Kirkpatrick, and D. R. Nelson, Phys. Rev. B 16, 1217 (1977).

${ }^{9}$ H. A. Fertig, Phys. Rev. Lett. 89, 035703 (2002).

${ }^{10}$ See article by S. M. Girvin and A. H. MacDonald in Perspectives on Quantum Hall Effects, S. Das Sarma and Aron Pinczuk, eds. (John Wiley and Sons, New York, 1997).

${ }^{11}$ R. Rajaraman, Solitons and Instantons (North-Holland, New York, 1989).

${ }^{12}$ B. Müller, The Physics of the Quark-Gluon Plasma (Springer Verlag, New York, 1985).

${ }^{13}$ C. Vanderzande, Lattice Models of Polymers, (Cambridge University Press, New York, 1998).

${ }^{14}$ H. A. Fertig and Joseph P. Straley, Phys. Rev. B. 66, 201402(R) (2002).

${ }^{15}$ D. Ruelle, Statistical Mechanics: Rigorous Results (Addison-Wesley, Reading, MA, 1989).

16 A. Paramakenti, L. Balents, and M. P. A. Fisher, Phys. Rev. B 66, 054526 (2002).

17 J. Villain, J. Phys. 36, 581 (1975).

${ }^{18}$ L. P. Kadanoff, Statistical Physics: Dynamics, Statics, and Renormalization (World Scientific, Singapore, 2000).

19 See article by J. D. Weeks in Ordering in Strongly Fluctuating Condensed Matter, T. Riste, ed. (Plenum Press, New York, 1979).

${ }^{20}$ In practice we will not need to enforce the boundary constraint, since relaxing it does not lead to spurious divergences in our RG analysis.

${ }^{21}$ N. D. Antunes, L. M. A. Bettencourt, and M. Kunz, condmat/0201149. 

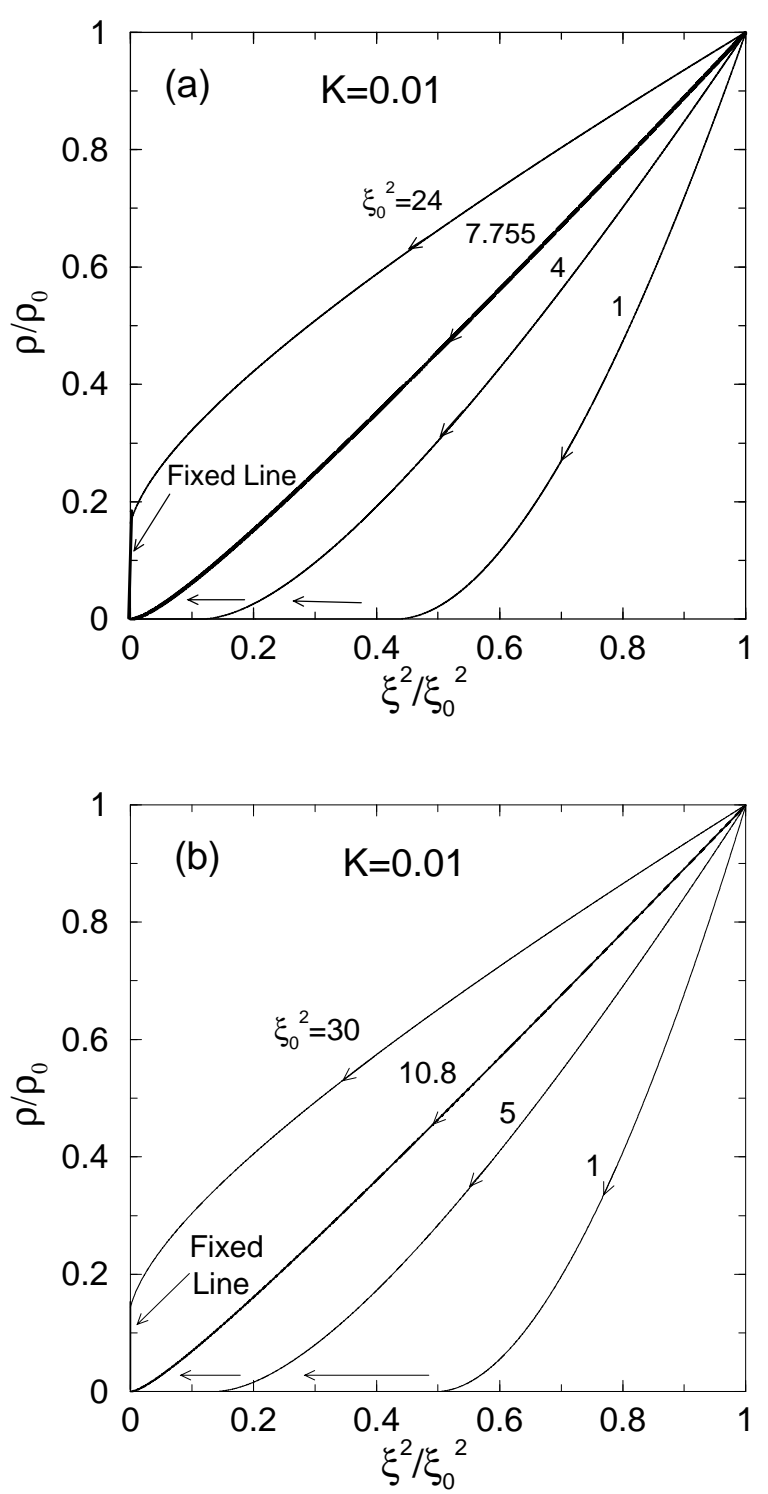

FIG. 10. RG flows for the scaling relation in Eq. 39 for (a) square lattice and (b) triangular lattice with full lattice symmetry. $K=0.01$ and initial $\rho_{0}=1 / 40$. Left vertical axis is a fixed line, and the thick line is a separatrix between flows that reach $\rho=0$ for finite $\ell$ and flow to the origin as $\ell \rightarrow \infty$, and those that have $\rho>0$ at the end of their flow. Thick lines are separatrices with critical values of (a) $\xi_{c r}^{2}=7.755$ and (b) $\xi_{c r}^{2}=10.8$.

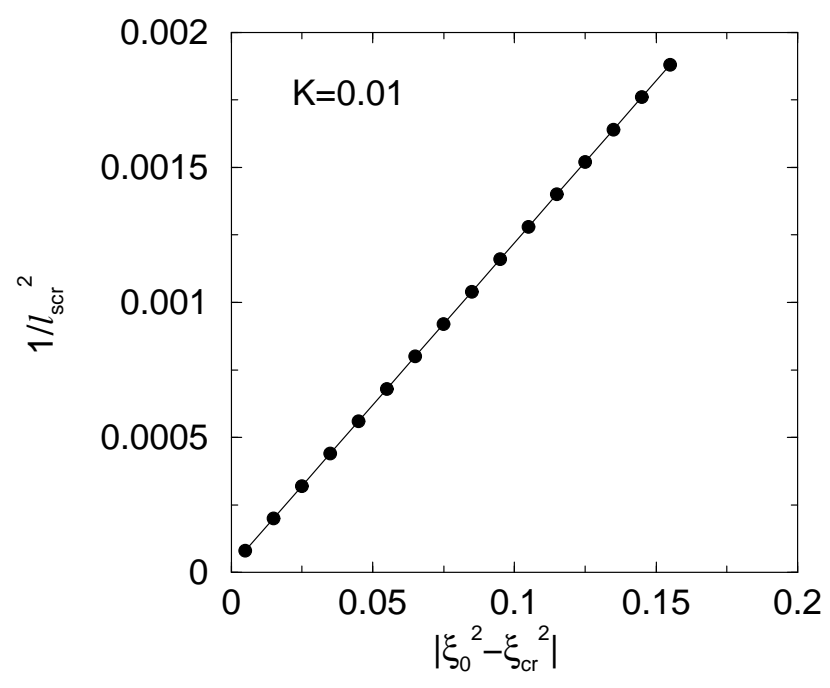

FIG. 11. $1 / \ell_{s c r}^{2}$ vs. $\left|\xi_{0}^{2}-\xi_{c r}^{2}\right|$ for square lattice with full symmetry where $\ell_{s c r}$ is the screening length. $\ell_{s c r}$ diverges with a critical exponent $-1 / 2$ as $\xi_{0}^{2} \rightarrow \xi_{c r}^{2}$ from below. Parameters are same as in Fig. 10.

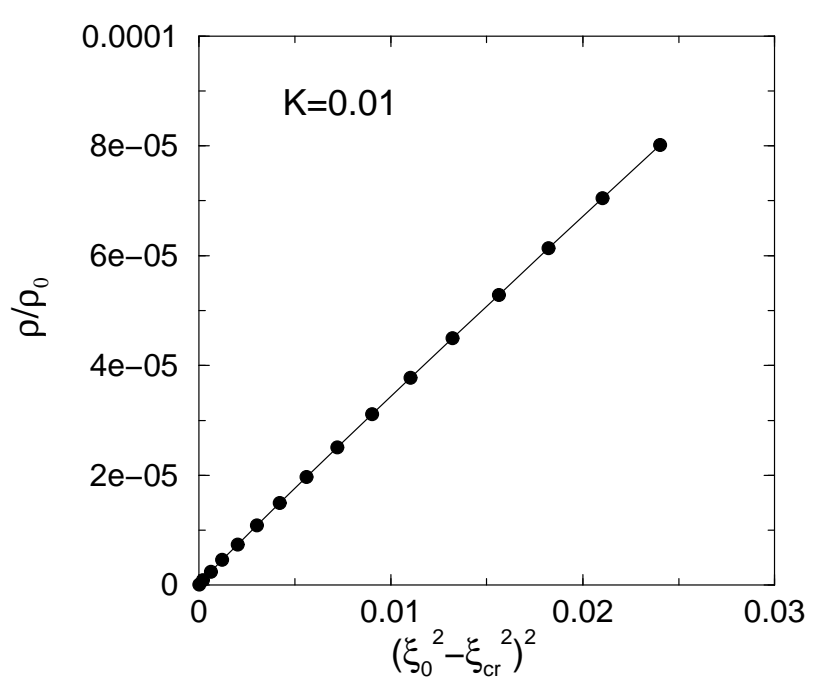

FIG. 12. Normalized string tension $\rho / \rho_{0}$ as a function of $\left(\xi_{0}^{2}-\xi_{c r}^{2}\right)^{2}$ for the square lattice with full lattice symmetry. $\rho$ vanishes quadratically as $\xi_{0}^{2} \rightarrow \xi_{c r}^{2}$ from above. Parameters are same as in Fig. 10. 